\title{
Stability with Large Step Sizes for Multistep Discretizations of Stiff Ordinary Differential Equations
}

\author{
By George Majda*
}

\begin{abstract}
In this paper we consider a large set of variable coefficient linear systems of ordinary differential equations which possess two different time scales, a slow one and a fast one. A small parameter $\varepsilon$ characterizes the stiffness of these systems. We approximate a system of ODE's in this set by a general class of multistep discretizations which includes both one-leg and linear multistep methods. We determine sufficient conditions under which each solution of a multistep method is uniformly bounded, with a bound which is independent of the stiffness of the system of ODE's, when the step size resolves the slow time scale but not the fast one. We call this property stability with large step sizes.

The theory presented in this paper lets us compare properties of one-leg methods and linear multistep methods when they approximate variable coefficient systems of stiff ODE's. In particular, we show that one-leg methods have better stability properties with large step sizes than their linear multistep counterparts. This observation is consistent with results obtained by Dahlquist and Lindberg [11], Nevanlinna and Liniger [33] and van Veldhuizen [42]. Our theory also allows us to relate the concept of $D$-stability (van Veldhuizen [42]) to the usual notions of stability and stability domains and to the propagation of errors for multistep methods which use large step sizes.
\end{abstract}

1. Introduction. Model equations (usually obtained through a linearization procedure) have played an extremely important role in assessing the behavior of a discretization of a system of stiff differential equations. The test equation,

$$
d y / d t=\lambda y, \quad \lambda \in \mathbf{C} \text { is a constant, }
$$

has led to the concepts of $A$-stability [7], $A(\alpha)$-stability [44], stiff-stability [18] and $L$-stability [13]. However, this model is too simple. Examples due to Gourlay [19] show that two different one-step methods with the same domain of absolute stability can have entirely different properties when they approximate the variable-coefficient test equation

$$
d y / d t=\lambda(t) y, \quad \lambda(t) \in \mathbf{C} \text { for } 0 \leqslant t \leqslant T .
$$

For this reason and others, Prothero and Robinson [36] introduced a theory for the stability and accuracy of one-step discretizations of stiff ODE's based on the

Received January 30, 1984; revised March 11, 1985.

1980 Mathematics Subject Classification. Primary 65L05, 65L20.

* Part of this research was done while the author was in residence at the Institute for Computer Applications in Science and Engineering, NASA Langley Research Center, Hampton, Virginia 23665. This work was supported under contract No. NAS1-17070.

This research was partially supported by the Office of Naval Research under grant No. N00014-85-K0620. 
model equation

$$
d y / d t=g^{\prime}(t)+\lambda(y-g(t)),
$$

where $\lambda$ is a complex constant with negative real part, ()$^{\prime}$ denotes the derivative with respect to $t$, and $g^{\prime}(t)$ is any function that is defined and bounded for $t \in[0, T]$ where $T$ is some constant.

In several papers nonlinear systems of ODE's which satisfy a monotonicity condition have been used for model problems. See Dahlquist [8], Odeh and Liniger [35], Stetter [38], Burrage [3], Burrage and Butcher [4], Butcher [5], and Crouzeix [6].

In [4] and [17], the authors introduced the concepts of $B$-stability and $B$-convergence for discretizations of nonautonomous systems of ODE's which satisfy a one-sided Lipschitz condition.

Most stiff systems, for example, the equations which arise in chemistry or electric circuit theory, have several components which respond with widely differing time constants. Furthermore, these components are usually coupled and both the coupling and the time constants are time dependent (cf. Bjurel et al. [2], Lapidus and Schiesser [25], or Willoughby [45]). Models (1.1)-(1.3), equations with monotone nonlinearities and an arbitrary class of equations which satisfy a one-sided Lipschitz condition do not adequately account for all these characteristics. Consequently, several authors, including Dahlquist [9], Miranker [31], [32], van Veldhuizen [40], [41], [42], and Stetter [37] have proposed more refined model equations for discretizations of stiff ODE's. Stetter suggested that an appropriate model equation should possess the following properties:

(1) It should permit the simultaneous occurrence of slowly varying and rapidly varying solution components.

(2) It should have a Jacobian matrix with a time-dependent eigensystem.

(3) It should contain a small parameter to permit the consideration of a limit process corresponding to a transition to arbitrarily high stiffness.

In this paper we will consider model linear systems of ODE's which satisfy properties (1)-(3). These equations are given by

$$
d v / d t=A(t, \varepsilon) v, \quad 0 \leqslant t \leqslant T, 0<\varepsilon \leqslant \varepsilon_{0}, v(0) \text { given, }
$$

and

$$
d y / d t=A(t, \varepsilon) y+F(t, \varepsilon), \quad 0 \leqslant t \leqslant T, 0<\varepsilon \leqslant \varepsilon_{0}, y(0) \text { given, }
$$

where $A(t, \varepsilon)$ is an $n \times n$ matrix, $v, y$, and $F(t, \varepsilon)$ are $n$-component vectors, and $T$, $\varepsilon_{0}$ are positive constants with $\varepsilon_{0} \ll 1 . A(t, \varepsilon)$ and $F(t, \varepsilon)$ should satisfy the conditions in the following three assumptions:

Assumption 1.1. There exists an invertible matrix $T(t, \varepsilon)$ with $T(t, \varepsilon), T^{-1}(t, \varepsilon) \in$ $C^{p}(t, \varepsilon, B),{ }^{* *}$ and $p \geqslant 3$ so that

$$
T^{-1}(t, \varepsilon) A(t, \varepsilon) T(t, \varepsilon)=\left(\begin{array}{cc}
D_{11}(t, \varepsilon) / \varepsilon & 0 \\
0 & D_{22}(t, \varepsilon)
\end{array}\right)=D(t, \varepsilon) .
$$

Here $D_{11}(t, \varepsilon) \in C^{p}(t, \varepsilon, B)$ is an $m \times m$ matrix with $1 \leqslant m<n$, and $D_{22}(t, \varepsilon) \in$ $C^{p}(t, \varepsilon, B)$ is an $(n-m) \times(n-m)$ matrix.

\footnotetext{
** All notation is defined in Appendix I.
} 
Assumption 1.2. $D_{11}(t, \varepsilon)$ is an invertible matrix for $0 \leqslant t \leqslant T$ and $0 \leqslant \varepsilon \leqslant \varepsilon_{0}$ with $D_{11}^{-1}(t, \varepsilon) \in C^{p}(t, \varepsilon, B)$. Furthermore, the eigenvalues of $D_{11}(t, \varepsilon)$, denoted by $\lambda_{i}(t, \varepsilon), i=1, \ldots, m$, satisfy $\operatorname{Re}\left\{\lambda_{i}(t, \varepsilon)\right\} \leqslant 0, i=1, \ldots, m$, and are distinct for $0 \leqslant t \leqslant T$ and $0 \leqslant \varepsilon \leqslant \varepsilon_{0}$.

Assumption 1.3. $\quad T^{-1}(t, \varepsilon) F(t, \varepsilon)=\left(f_{\mathrm{I}}(t, \varepsilon) / \varepsilon, f_{\mathrm{II}}(t, \varepsilon)\right)^{T}, \quad$ where $f_{\mathrm{I}}(t, \varepsilon) \in$ $C^{p}(t, \varepsilon, B)$ denotes an $m$-component vector and $f_{\mathrm{II}}(t, \varepsilon) \in C^{p}(t, \varepsilon, B)$ denotes an $(n-m)$-component vector.

An asymptotic decomposition for the solutions of systems (1.5) is easily obtained. The results in Hoppensteadt [20], Hoppensteadt and Miranker [21], and Kreiss [23] imply that if $\varepsilon$ is sufficiently small, then the solutions of systems (1.5) which satisfy the conditions in Assumptions 1.1-1.3 have an additive decomposition with the form

$$
y(t, \varepsilon)=y^{s}(t, \varepsilon)+y^{R}(\tau, \varepsilon) \quad \text { with } \tau=t / \varepsilon .
$$

$y^{s}(t, \varepsilon)$ is a slowly varying solution of $(1.5)$ with $(p-2)$ derivatives with respect to $t$ bounded independently of $\varepsilon \cdot y^{R}(\tau, \varepsilon)$ is a rapidly varying solution of (1.5) which is highly oscillatory (rapidly decaying) if $\operatorname{Re}\left\{\lambda_{i}(t, \varepsilon)\right\}=0, i=1, \ldots, m,\left(\operatorname{Re}\left\{\lambda_{i}(t, \varepsilon)\right\}\right.$ $<0, i=1, \ldots, m)$. Both $y^{s}(t, \varepsilon)$ and $y^{R}(\tau, \varepsilon)$ are uniquely determined up to terms $O\left(\varepsilon^{(p-2)}\right)$.

In order to compare the properties of various multistep methods, we will consider the general class of multistep approximations of (1.4) and (1.5) given by

$$
\sum_{i=0}^{r}\left(a_{i} I+k b_{i} A\left(\tau_{i}\left(t_{n}\right), \varepsilon\right)\right) v^{n-i}=0, \quad n=r, \ldots, N
$$

and

$$
\begin{aligned}
\sum_{i=0}^{r}\left(a_{i} I+k b_{i} A\left(\tau_{i}\left(t_{n}\right), \varepsilon\right)\right) y^{n-i}+k \mathscr{F}\left(\tau_{0}\left(t_{n}\right), \ldots, \tau_{r}\left(t_{n}\right), k, \varepsilon\right) & =0, \\
n & =r, \ldots, N .
\end{aligned}
$$

Here $a_{i}, b_{i}, i=0, \ldots, r$, denote given constants, $k>0$ denotes a constant step size, $t_{n}=n k, \quad n=0,1, \ldots, N, \tau_{i}(t) \in C^{1}(t, B), \quad i=0, \ldots, r, \tau_{i}(t)=\tau_{0}(t)+O(k)$ for $i=1, \ldots, r$ and $0 \leqslant t \leqslant T, 0 \leqslant \tau_{i}(t) \leqslant T$ for $i=0, \ldots, r$ and $t_{r} \leqslant t \leqslant T$, and $\mathscr{F}\left(\tau_{0}(t), \ldots, \tau_{r}(t), k, \varepsilon\right)$ denotes a function which depends on $F\left(\tau_{i}(t), \varepsilon\right), i=0, \ldots, r$. We will assume that $\mathscr{F} \equiv 0$ when $F(t, \varepsilon) \equiv 0$ and that the coefficients in (1.8) and (1.9) are normalized so that $a_{0}=1$. We will refer to method (1.8) as the homogeneous multistep method corresponding to method (1.9).

Under special conditions, methods (1.9) lead to well-known classes of multistep methods. If we set $\tau_{i}(t)=t-i k, i=0, \ldots, r$, and $\mathscr{F}=\sum_{i=0}^{r} b_{i} F(t-i k, \varepsilon)$, then (1.9) becomes

$$
\sum_{i=0}^{r}\left(a_{i} I+k b_{i} A\left(t_{n-i}, \varepsilon\right)\right) y^{n-i}+k \sum_{i=0}^{r} b_{i} F\left(t_{n-i}, \varepsilon\right)=0
$$

which is a linear multistep method. On the other hand, if we set $\tau_{i}(t)=\tau_{0}(t)=$ $\sum_{j=0}^{r} b_{j}(t-j k), i=0, \ldots, r$, and $\mathscr{F}=F\left(\tau_{0}(t), \varepsilon\right)$, then $(1.9)$ becomes

$$
\sum_{i=0}^{r}\left(a_{i} I+k b_{i} A\left(\sum_{j=0}^{r} b_{j} t_{n-j}, \varepsilon\right)\right) y^{n-i}+k F\left(\sum_{j=0}^{r} b_{j} t_{n-j}, \varepsilon\right)=0
$$

which is a one-leg method. These methods were introduced by Dahlquist [8]. 
In this paper we are particularly interested in the properties of discretizations (1.8) and (1.9) when the step size $k$ resolves only the slow time scales in system (1.4) or (1.5) and not the fast ones. This is the typical situation for discretizations of stiff equations. Therefore, we are interested in the cases when $\varepsilon$ tends to zero for fixed $k>0$ or $k \rightarrow 0$ with $k / \varepsilon$ bounded away from zero. (The limit process $k / \varepsilon \rightarrow 0$ would take us back to the well-known Dahlquist-Henrici theory of discretizations on "sufficiently fine" grids.) Consequently, the two parameters $\varepsilon$ and $k$ will vary in the trapezoidal region

$$
\Gamma=\left\{(k, \varepsilon) \in \mathbf{R}^{2}: 0<\varepsilon \leqslant \varepsilon_{0} \text { and } \sigma^{*} \varepsilon \leqslant k \leqslant k_{0}\right\},
$$

where $\sigma^{*}$ and $k_{0}$ are two positive constants (see Figure 1).

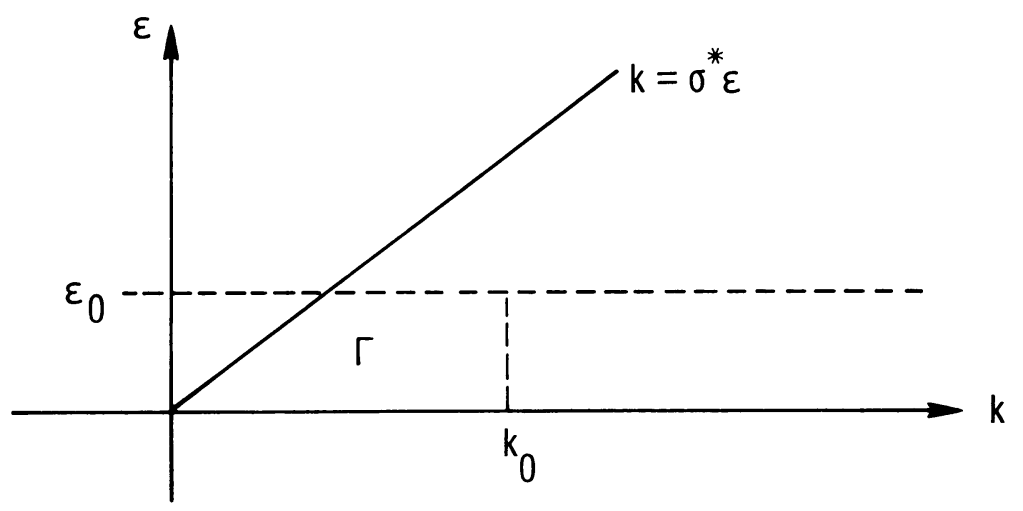

FIGURE 1

Representation of the trapezoidal region $\Gamma$.

Following the ideas and results in van Veldhuizen [40], [41], Stetter [37] suggested that a desirable discretization of a system (1.5) should satisfy two properties. First, each solution of the numerical method should be uniformly bounded with a bound which is independent of the stiffness of the equation. We formulate this requirement as

Property 1.1. For some trapezoidal region $\Gamma$, each solution of (1.9), denoted by $y^{n}, n=0, \ldots, N$, should be uniformly bounded for all $(k, \varepsilon) \in \Gamma$.

A multistep approximation of a system (1.5) which satisfies Property 1.1 is called a stable approximation of (1.5) with large step sizes.

The second property pertains to the accuracy of the numerical solution. In particular, only the slow time scales in (1.5) are resolved by the step size while the fast scales are not resolved. Therefore, the numerical approximation of (1.5) should have a two-term decomposition corresponding to (1.7). The first term should approximate the slowly varying solution $y^{s}(t, \varepsilon)$ in the decomposition (1.7) with some degree of accuracy. The second term should decay with time. This behavior of the second term is consistent with the properties of $y^{R}(\tau, \varepsilon)$ when $y^{R}(\tau, \varepsilon)$ is rapidly decaying. In the case when $y^{R}(\tau, \varepsilon)$ contains rapidly oscillating components, this restriction assumes that the rapidly oscillating components have no interest and should be attenuated. We formulate this accuracy requirement as

Property 1.2. For each value $n=0,1, \ldots, N, y^{n}$ should have the decomposition

$$
y^{n}=y_{s}^{n}+E^{n} \text {, }
$$


where $y_{s}^{n}$ approximates $y^{s}\left(t_{n}, \varepsilon\right)$ uniformly well for all $(k, \varepsilon) \in \Gamma$, that is,

$$
\left\|y_{s}^{n}-y^{s}\left(t_{n}, \varepsilon\right)\right\| \leqslant K_{1}\left(k^{q_{1}}+\varepsilon^{q_{2}}\right) \quad \text { when }(k, \varepsilon) \in \Gamma,
$$

where $K_{1}$ is some constant independent of $k$ and $\varepsilon$ and $q_{1}, q_{2}$ are two positive integers. $E^{n}$ should decay, that is,

$$
\left\|E^{n}\right\| \leqslant K_{2} \delta^{n} \quad \text { when }(k, \varepsilon) \in \Gamma
$$

where $K_{2}$ and $0 \leqslant \delta<1$ are constants independent of $k$ and $\varepsilon$.

In this paper, we apply the concept of stability domains together with the usual Dahlquist-Henrici stability theory to determine sufficient conditions under which a multistep approximation of (1.5) with the form (1.9) satisfies Property 1.1. One immediate consequence of this theory is that one-leg methods have better stability properties with large step sizes than their linear multistep counterparts when they approximate variable coefficient (and nonlinear) systems of stiff ordinary differential equations. A second consequence of our theory is to relate the concept of $D$-stability (van Veldhuizen [42]) to the usual notions of stability and stability domains and to the propagation of errors for multistep methods which use large step sizes. Additional consequences are discussed in Sections 3 and 5. We determine sufficient conditions under which a multistep approximation of (1.5) satisfies Property 1.2 in a forthcoming paper using the techniques developed in [29].

The results in this paper either extend or complement known results in several ways. Van Veldhuizen [40], [41], established Properties 1.1 and 1.2 for a class of one-step methods using techniques which are entirely different from the ones presented in this paper. He was unable to extend his theory to multistep methods. Dahlquist and Lindberg [11] compared the stability, accuracy, and step changing properties of the implicit midpoint rule and the trapezoidal rule when these methods approximate stiff linear and nonlinear systems of ODE's. Kreiss [22] essentially determines sufficient conditions under which $A(\alpha)$-stable linear multistep approximations of systems (1.4) satisfy Property 1.1 when the matrix $A(t, \varepsilon)$ in $(1.4)$ is of essentially diagonally dominant type. Kreiss's results cannot be extended to the class of systems (1.4) which satisfy Assumptions 1.1 and 1.2. (See van Veldhuizen [42] for details.) Dahlquist and Söderland [12] essentially establish Property 1.1 for a class of multistep and multistep compound discretizations of systems (1.5) which appear in singular perturbation form using the theory of $G$-contractivity. The systems (1.5) which we consider do not appear in singular perturbation form. Furthermore, we consider a class of multistep discretizations of (1.5) which is larger than the class of multistep discretizations considered by Dahlquist and Söderland. Finally, Abrahamsson, Keller, and Kreiss [1] have established Properties 1.1 and 1.2 for a class of three-point difference approximations of some linear two-point boundary value problems which appear in singular perturbation form.

This paper is organized in the following way: In Section 2 we consider multistep approximations of the special systems $\varepsilon d y / d t=D_{11}(t, \varepsilon) y$, where $D_{11}(t, \varepsilon)$ satisfies the conditions in Assumption 1.2. These systems govern the rapidly varying components of systems (1.4) and (1.5). We use properties of stability domains to determine a trapezoidal region $\Gamma$ with the form (1.12), so that all solutions of a multistep approximation of these special systems decay with time when $(k, \varepsilon) \in \Gamma$. In Section 3 we determine sufficient conditions under which a multistep approximation of 
system (1.5) satisfies Property 1.1. We also present a thorough discussion of the practical implications of Property 1.1 for a multistep method. In Section 4 we relate the concept of $D$-stability (van Veldhuizen [42]) to the results obtained in Section 3. In Section 5 we present some numerical results.

2. Multistep Approximations of the Unresolved Fast Scales. In this section we will consider multistep approximations of the special systems

$$
\varepsilon \frac{d w}{d t}=D_{11}(t, \varepsilon) w, \quad 0 \leqslant t \leqslant T, 0<\varepsilon \leqslant \varepsilon_{0}, w(0) \text { given, }
$$

where $T$ and $\varepsilon_{0} \ll 1$ are given constants and $D_{11}(t, \varepsilon) \in C^{p}(t, \varepsilon, B)$ with $p \geqslant 1$ is an $m \times m$ matrix which satisfies the conditions in Assumption 1.2. Systems (2.1) govern the rapidly varying components of systems (1.4) and (1.5). In particular, the results in [16] or [43] show that if $\varepsilon_{0}$ is sufficiently small, then for any integer $0 \leqslant \nu \leqslant p-1$ the solutions of (2.1) have an asymptotic decomposition with the form

$$
\begin{array}{r}
w(t)=\operatorname{diag}\left(e^{\int_{0}^{t} \beta_{1}(\gamma, \varepsilon) d \gamma}, \ldots, e^{\int_{0}^{t} \beta_{m}(\gamma, \varepsilon) d \gamma}\right) w(0)+O\left(\varepsilon^{\nu}\right) \\
\text { for } 0 \leqslant t \leqslant T \text { and } 0<\varepsilon \leqslant \varepsilon_{0},
\end{array}
$$

where

$$
\beta_{i}(t, \varepsilon)=\frac{\lambda_{i}(t, 0)}{\varepsilon}+O(1) \text { for } i=1, \ldots, m, 0 \leqslant t \leqslant T, \text { and } 0<\varepsilon \leqslant \varepsilon_{0} .
$$

Consequently, the solutions of (2.1) are highly oscillatory (rapidly decaying) if $\operatorname{Re}\left\{\lambda_{i}(t, \varepsilon)\right\}=0, i=1, \ldots, m\left(\operatorname{Re}\left\{\lambda_{i}(t, \varepsilon)\right\}<0, i=1, \ldots, m\right)$.

We will consider multistep approximations of (2.1) with the form (1.8) using a fixed step size $k$ which is bounded but does not resolve the rapidly varying components, that is, $k$ and $\varepsilon$ satisfy $k \leqslant k_{0}$ and $k / \varepsilon \gg 1$, where $k_{0}$ is some constant. We use properties of stability domains to determine sufficient conditions under which all solutions of a multistep approximation of (2.1) decay with time when $k$ and $\varepsilon$ satisfy the previously stated conditions. This behavior of the solution of the multistep method is consistent with the properties of the solutions of (2.1) when the solutions of (2.1) decay. In the case when the solutions of (2.1) contain rapidly oscillating components, this restriction assumes that the rapidly oscillating components have no interest and should be attenuated.

In order to state our main result, we need to define the usual notion of a domain of absolute stability for a multistep method (1.9), and to introduce two concepts which generalize the notions of $A$-stability and $L$-stability to subsets of the complex plane other than the left half-plane.

Approximate the test equation (1.1) by a multistep method (1.9) and set $z=k \lambda$ to obtain the difference equation

$$
\sum_{i=0}^{r}\left(a_{i}+b_{i} z\right) w^{n-i}=0, \quad r \leqslant n<\infty .
$$

Set $\tilde{w}^{n}=\left(w^{n}, \ldots, w^{n-r+1}\right)^{T}$ for $r-1 \leqslant n \leqslant \infty$ and let $\Omega$ denote a subset of the complex plane.

Definition 2.1. (a) The domain of absolute stability of (1.9) consists of all points $z \in \mathbf{C}$ such that $\left(a_{0}+b_{0} z\right)^{-1}$ exists, and there exists a constant $K(z)$ so that all 
solutions of (2.2) satisfy

$$
\left\|\tilde{w}^{n}\right\| \leqslant K(z)\left\|\tilde{w}^{j}\right\| \text { for } r-1 \leqslant j \leqslant n<\infty .
$$

(b) The multistep method (1.9) is strictly $\Omega$-stable if $\left(a_{0}+b_{0} z\right)^{-1}$ exists for all $z \in \Omega$, and for each $z \in \Omega$ there exist constants $K(z)$ and $\delta(z)$ with $0 \leqslant \delta(z)<1$ so that all solutions of (2.2) satisfy

$$
\left\|\tilde{w}^{n}\right\| \leqslant K(z)(\delta(z))^{n-j}\left\|\tilde{w}^{j}\right\| \text { for } r-1 \leqslant j \leqslant n<\infty .
$$

(c) The multistep method (1.9) is strongly $\Omega$-stable if

$$
\left(a_{0}+b_{0} z\right)^{-1} \text { exists and is uniformly bounded for all } z \in \Omega \text {, }
$$

and there exist constants $K>0$ and $0 \leqslant \delta<1$, independent of $z$, so that all solutions of (2.2) satisfy

$$
\left\|\tilde{w}^{n}\right\| \leqslant K \delta^{n-j}\left\|\tilde{w}^{j}\right\| \text { for } r-1 \leqslant j \leqslant n<\infty \text { and all } z \in \Omega .
$$

Algebraic conditions which determine the domain of absolute stability of a multistep method, or subsets $\Omega$ in which a multistep method is either strictly or strongly $\Omega$-stable, are easily stated.

The solutions of the difference equation (2.2) are determined by the roots of the polynomial $R(z, \kappa)=\sum_{i=0}^{r}\left(a_{i}+b_{i} z\right) \kappa^{r-i}$ which we denote by $\kappa_{i}(z), i=1, \ldots, r$.

Definition 2.2. The roots of a polynomial $q(x)$ satisfy the root condition if all roots of $q(x)=0$, denoted by $x_{\nu}, \nu=1, \ldots, Q$, satisfy $\left|x_{\nu}\right| \leqslant 1, \nu=1, \ldots, Q$, and those roots which lie on the unit circle are simple.

LEMMA 2.1. (a) The domain of absolute stability for method (1.9) consists of all points $z \in \mathbf{C}$ such that $\left(a_{0}+b_{0} z\right)^{-1}$ exists and the roots of $R(z, \kappa)$ satisfy the root condition.

(b) The multistep method (1.9) is strictly $\Omega$-stable if $\left(a_{0}+b_{0} z\right)^{-1}$ exists for all $z \in \Omega$ and the roots of $R(z, \kappa)$ satisfy $\left|\kappa_{i}(z)\right|<1, i=1, \ldots, r$.

(c) The multistep method (1.9) is strongly $\Omega$-stable if condition (2.3) is satisfied and there exists a constant $0 \leqslant \gamma<1$, independent of $z$, so that the roots of $R(z, \kappa)$ satisfy $\left|\kappa_{i}(z)\right|<\gamma$ for $i=1, \ldots, r$, and all $z \in \Omega$.

Example 2.1. Consider the trapezoidal approximation of (1.5) given by

$$
y^{n}=y^{n-1}+\frac{k}{2}\left(A\left(t_{n}, \varepsilon\right) y^{n}+A\left(t_{n-1}, \varepsilon\right) y^{n-1}\right)+\frac{k}{2}\left(F\left(t_{n}, \varepsilon\right)+F\left(t_{n-1}, \varepsilon\right)\right) \text {. }
$$

Set $D=\{(x+i y) \in \mathbf{C}: x \leqslant 0\}$ and $D^{\prime}=\{(x+i y) \in \mathbf{C}: x<0\}$. Then

(i) The domain of absolute stability of the trapezoidal rule is the set $D$.

(ii) The trapezoidal rule is strictly $D^{\prime}$-stable but not strongly $D^{\prime}$-stable.

Identical remarks apply to the implicit midpoint rule

$$
y^{n}=y^{n-1}+\frac{k}{2} A\left(t_{n-1 / 2}, \varepsilon\right)\left(y^{n}+y^{n-1}\right)+k F\left(t_{n-1 / 2}, \varepsilon\right),
$$

which is the one-leg twin associated with the trapezoidal rule.

Example 2.2. For each value of $p=1, \ldots, 6$, the backward-difference formula of order $p\left(\left[24\right.\right.$, p. 242], or [18, Chapter 11]) is strongly $\Omega_{p}$-stable in the region

$$
\Omega_{p}=\left\{z \in \mathrm{C}:-\alpha_{p}+\varepsilon \leqslant \pi-\arg (z) \leqslant \alpha_{p}-\varepsilon \text { and }|z|>\rho\right\},
$$


where $\rho$ is any positive constant and $\varepsilon$ is any constant satisfying $0<\varepsilon \leqslant \alpha_{p}$ with $\alpha_{1}=90^{\circ}, \alpha_{2}=90^{\circ}, \alpha_{3}=88^{\circ}, \alpha_{4}=73^{\circ}, \alpha_{5}=51^{\circ}$, and $\alpha_{6}=18^{\circ}$.

If we set $\rho=0$ in (2.4), then each backward-difference formula of order $p$ is strictly $\Omega_{p}$-stable but not strongly $\Omega_{p}$-stable.

We are now ready to state the main result of this section.

THEOREM 2.1. Approximate system (2.1) by a multistep method (1.8) with a step size $k$ satisfying $0<k \leqslant \bar{k}$, where $\bar{k}$ is some constant, and set $\tilde{y}^{n}=\left(y^{n}, \ldots, y^{n-r+1}\right)^{T}$. Let $\Omega$ denote a subset of the complex plane. Assume that the multistep method is implicit, strongly $\Omega$-stable, and there exists a trapezoidal region $\Gamma^{\prime}$ with the form

$$
\Gamma^{\prime}=\left\{(k, \varepsilon) \in \mathbf{R}^{2}: 0<\varepsilon \leqslant \varepsilon_{0} \text { and } \sigma^{*} \varepsilon \leqslant k \leqslant \bar{k}\right\},
$$

where $\sigma^{*}>0$ is some constant such that

$$
(k / \varepsilon) \lambda_{i}(t, \varepsilon) \in \Omega \quad \text { for } i=1, \ldots, m, 0 \leqslant t \leqslant T \text {, and }(k, \varepsilon) \in \Gamma^{\prime} .
$$

Then, if $\varepsilon_{0}$ is sufficiently small, there exist constants $K>0,0 \leqslant \delta<1$, and $k_{0} \leqslant \bar{k}$, independent of $t, k$, and $\varepsilon$, such that all solutions of (1.8) satisfy the estimate

$$
\left\|\tilde{y}^{n}\right\| \leqslant K \delta^{n-j}\left\|\tilde{y}^{j}\right\| \quad \text { for all } r-1 \leqslant j \leqslant n \leqslant N \text { and }(k, \varepsilon) \in \Gamma,
$$

where $\Gamma$ is the set defined on line (1.12).

We make several remarks pertaining to the hypotheses in Theorem 2.1 before presenting its proof.

(1) If condition (2.6) is satisfied for all $0<\varepsilon \leqslant \varepsilon_{0}$, then the set $\Omega$ must contain the point at $\infty$.

(2) If $\Omega$ is star-shaped at $\infty$ (i.e., $z \in \Omega$ implies that $c z \in \Omega$ for all $1 \leqslant c<\infty$ ), and the eigenvalues of $D_{11}(t, \varepsilon)$ lie in $\Omega$, that is, $\lambda_{i}(t, \varepsilon) \in \Omega$ for $i=1, \ldots, m$, $0 \leqslant t \leqslant T$, and $0<\varepsilon \leqslant \varepsilon_{0}$, then there exist constants $\bar{k}, \sigma^{*}$ and a trapezoidal region $\Gamma^{\prime}$ so that condition (2.6) is satisfied. In this case, set $\sigma^{*}=1$ and let $\bar{k}$ be any constant satisfying $\bar{k} \geqslant \varepsilon_{0}$.

(3) The condition that $k_{0} \leqslant \bar{k}$ is natural in order to account for the rate of change of the eigenvectors of the matrix $D_{11}(t, \varepsilon)$ which one misses by only examining the class of scalar test equations (1.1).

(4) If the multistep method satisfies the conditions in Theorem 2.1 but is strictly $\Omega$-stable and not strongly $\Omega$-stable, then estimate (2.7) does not hold. Approximate the equation $\dot{y}=-(1 / \varepsilon) y$ by the trapezoidal rule to justify this comment.

The proof of Theorem 2.1 is a direct application of the following lemma which relates the behavior of a multistep approximation of a system of ODE's with variable coefficients to the behavior of the multistep approximation for a class of scalar test problems (1.1).

Lemma 2.2. Let $\Gamma^{\prime}$ denote the set defined on line (2.5). Consider the difference equation

$$
y^{n}=\sum_{j=1}^{r} Q_{j}\left(k, \varepsilon, \tau_{0}\left(t_{n}\right), \ldots, \tau_{r}\left(t_{n}\right)\right) y^{n-j}
$$

$$
\text { for } n=r, r+1, \ldots, \text { and }(k, \varepsilon) \in \Gamma^{\prime},
$$

where $y^{n}$ is an m-component vector and each $Q_{j}, j=1, \ldots, r$, is an $m \times m$ matrix. 
Assume that the following two conditions are satisfied:

(1) There exists a constant $\bar{Q}$, independent of $t, k$, and $\varepsilon$, such that

$$
Q_{j}\left(k, \varepsilon, \tau_{0}(t), \ldots, \tau_{r}(t)\right) \in C^{1}(t, \bar{Q}) \quad \text { for } r k \leqslant t \leqslant \infty \text { and }(k, \varepsilon) \in \Gamma^{\prime}
$$

and

$$
\begin{array}{rl}
Q_{j}\left(k, \varepsilon, \tau_{0}(t), \ldots, \tau_{r}(t)\right)=Q_{j}\left(k, \varepsilon, \tau_{0}(t), \ldots, \tau_{0}(t)\right)+k E_{j}, & \\
j & j=1, \ldots, r,
\end{array}
$$

where each matrix $E_{j}, j=1, \ldots, r$, is uniformly bounded for all $r k \leqslant t \leqslant \infty$ and all $(k, \varepsilon) \in \Gamma^{\prime}$.

(2) For each point $t^{*}$ satisfying $r k \leqslant t^{*}<\infty$ there exist constants $\tilde{K}>0$ and $0 \leqslant \tilde{\delta}<1$, independent of $t^{*}, k$, and $\varepsilon$, so that the solutions of each "frozen coefficient" difference equation

$$
v^{n}=\sum_{j=1}^{r} Q_{j}\left(k, \varepsilon, \tau_{0}\left(t^{*}\right), \ldots, \tau_{0}\left(t^{*}\right)\right) v^{n-j}, \quad n=r, r+1, \ldots
$$

satisfy the estimate

$$
\left\|\tilde{v}^{n}\right\| \leqslant \tilde{K} \tilde{\delta}^{n-j}\left\|\tilde{v}^{j}\right\| \quad \text { with } \tilde{v}^{n}=\left(v^{n}, v^{n-1}, \ldots, v^{n-(r-1)}\right)^{T}
$$

for all $r-1 \leqslant j \leqslant n<\infty$ and $(k, \varepsilon) \in \Gamma^{\prime}$. Then, if $\varepsilon_{0}$ is sufficiently small, there exist constants $K>0,0 \leqslant \delta<1$, and $k_{0} \leqslant \bar{k}$, independent of $t, k$, and $\varepsilon$, such that the solutions of (2.8) satisfy an estimate of the form

$$
\left\|\tilde{y}^{n}\right\| \leqslant K \delta^{n-j}\left\|\tilde{y}^{j}\right\| \quad \text { with } \tilde{y}^{n}=\left(y^{n}, y^{n-1}, \ldots, y^{n-(r-1)}\right)^{T}
$$

for all $r-1 \leqslant j \leqslant n<\infty$ and $(k, \varepsilon) \in \Gamma$, where $\Gamma$ is the set defined on line (1.12).

The proof of this result is lengthy and appears in Appendix II in the supplements section at the end of this issue.

Proof of Theorem 2.1. The eigenvalues of the matrix $(k / \varepsilon) D_{11}\left(\tau_{0}(t), \varepsilon\right)$ are distinct and are contained in $\Omega$ for all $0 \leqslant t \leqslant T$ and $(k, \varepsilon) \in \Gamma^{\prime}$, and the multistep method is strongly $\Omega$-stable, so

$$
\begin{aligned}
& \left(a_{0} I+\left(\frac{b_{0} k}{\varepsilon}\right) D_{11}\left(\tau_{0}(t), \varepsilon\right)\right)^{-1} \quad \text { exists and is uniformly bounded for all } \\
& r k \leqslant t \leqslant T \text { and }(k, \varepsilon) \in \Gamma^{\prime} .
\end{aligned}
$$

Consequently, for all $(k, \varepsilon) \in \Gamma^{\prime}$, the multistep approximation of system (2.1) can be written as

$$
y^{n}=\sum_{j=1}^{r} R_{j}\left(\left(\frac{1}{\varepsilon}\right) D_{11}\left(\tau_{0}\left(t_{n}\right), \varepsilon\right),\left(\frac{1}{\varepsilon}\right) D_{11}\left(\tau_{j}\left(t_{n}\right), \varepsilon\right), k, \varepsilon\right) y^{n-j},
$$

$$
n=r, \ldots, N
$$

where

$$
\begin{array}{r}
R_{j}\left(\left(\frac{1}{\varepsilon}\right) D_{11}\left(\tau_{0}\left(t_{n}\right), \varepsilon\right),\left(\frac{1}{\varepsilon}\right) D_{11}\left(\tau_{j}\left(t_{n}\right), \varepsilon\right), k, \varepsilon\right) \\
=\left(a_{0} I+\left(\frac{b_{0} k}{\varepsilon}\right) D_{11}\left(\tau_{0}\left(t_{n}\right), \varepsilon\right)\right)^{-1}\left(a_{j} I+\left(\frac{b_{j} k}{\varepsilon}\right) D_{11}\left(\tau_{j}\left(t_{n}\right), \varepsilon\right)\right) \\
j=1, \ldots, r
\end{array}
$$


Simple calculations (see [28, Section 5] for details) establish that there exists a constant $\bar{R}$, independent of $k, t$, and $\varepsilon$, such that

$$
R_{j}\left(\left(\frac{1}{\varepsilon}\right) D_{11}\left(\tau_{0}(t), \varepsilon\right),\left(\frac{1}{\varepsilon}\right) D_{11}\left(\tau_{j}(t), \varepsilon\right)\right) \in C^{1}(t, \bar{R})
$$

and

$$
\begin{aligned}
& R_{j}\left(\left(\frac{1}{\varepsilon}\right)\right.\left.D_{11}\left(\tau_{0}(t), \varepsilon\right),\left(\frac{1}{\varepsilon}\right) D_{11}\left(\tau_{j}(t), \varepsilon\right)\right) \\
&=R_{j}\left(\left(\frac{1}{\varepsilon}\right) D_{11}\left(\tau_{0}(t), \varepsilon\right),\left(\frac{1}{\varepsilon}\right) D_{11}\left(\tau_{0}(t), \varepsilon\right)\right)+O(k)
\end{aligned}
$$

for all $r k \leqslant t \leqslant T$ and $(k, \varepsilon) \in \Gamma^{\prime}$.

Let $t^{*}$ be any point in $[0, T]$ and consider the "frozen coefficient" difference equation

$$
v^{n}=\sum_{j=1}^{r} R_{j}\left(\left(\frac{1}{\varepsilon}\right) D_{11}\left(t^{*}, \varepsilon\right),\left(\frac{1}{\varepsilon}\right) D_{11}\left(t^{*}, \varepsilon\right)\right) v^{n-j}, \quad n=r, \ldots, N
$$

The eigenvalues of $(k / \varepsilon) D_{11}\left(t^{*}, \varepsilon\right)$ are distinct and contained in $\Omega$ for all $(k, \varepsilon) \in \Gamma^{\prime}$, and the multistep method is strongly $\Omega$-stable, so the solutions of (2.19) satisfy an estimate of the form (2.12) for all $t^{*} \in[0, T]$.

Since $0 \leqslant \tau_{0}\left(t^{*}\right) \leqslant T$ for $r k \leqslant t^{*} \leqslant T$, the solutions of (2.11) satisfy an estimate of the form (2.12) for all $t^{*} \in[r k, T]$.

A direct application of Lemma 2.2 completes the proof of Theorem 2.1.

Remark 2.1. If $D_{11}(t, \varepsilon) \in C^{p}(t, \varepsilon, B)$ for all $0 \leqslant t<\infty$ and $0 \leqslant \varepsilon \leqslant \varepsilon_{0}$ and satisfies the conditions in Assumption 1.2 for all $0 \leqslant t<\infty$ and $0 \leqslant \varepsilon \leqslant \varepsilon_{0}$, and the matrix $U(t, \varepsilon)$ which diagonalizes $D_{11}(t, \varepsilon)$ satisfies

$$
\sup _{\substack{0 \leqslant t<\infty \\ 0 \leqslant \varepsilon \leqslant \varepsilon_{0}}}\left\{\|U(t, \varepsilon)\|,\left\|U^{-1}(t, \varepsilon)\right\|\right\} \leqslant C,
$$

where $C$ is some constant, then estimate (2.7) holds for all $j$ and $n$ satisfying $r-1 \leqslant j \leqslant n<\infty$.

Remark 2.2. A result in the spirit of this section was derived by Odeh and Liniger in Section III of [35]. They considered one-leg approximations of nonlinear systems of ODE's which satisfy a monotonicity condition. They showed that if the one-leg method is $A_{\infty}$-stable (Odeh and Liniger [34]), then errors produced by initial data perturbations for the one-leg method decay exponentially with time. Analogous results were obtained for linear multistep methods by using the nonlinear transformation which relates the solution of a one-leg method to its corresponding linear multistep method.

The results of Liniger and Odeh cannot be used to obtain the result in this section because we consider systems (2.1) which do not necessarily satisfy the stated monotonicity condition, and we consider a class of multistep approximations of systems (2.1) which is larger than the union of the one-leg and linear multistep methods. Even if we restrict our discussion to systems (2.1) which satisfy the stated monotonicity condition for each fixed $0<\varepsilon \leqslant \varepsilon_{0}$, and to the classes of one-leg and linear multistep methods, the results of Liniger and Odeh are still inapplicable in this section. The transformation which connects the solution of a linear multistep 
approximation of (2.1) to the corresponding one-leg approximation of (2.1) is unbounded as $\varepsilon \rightarrow 0$ (van Veldhuizen [42]).

3. Property 1.1. In this section, we apply the concept of stability domains together with the usual Dahlquist-Henrici stability theory to determine sufficient conditions under which a multistep approximation of (1.5) with the form (1.9) satisfies Property 1.1 .

Remark 3.1. Throughout Section 3, $\Gamma$ will denote the trapezoidal region defined on line (1.12); we will assume that all constants are independent of the parameters $k$, $t$, and $\varepsilon$ unless explicitly stated otherwise, and whenever we refer to systems (1.4) or (1.5), we will always assume that these systems satisfy the conditions in Assumptions 1.1-1.3.

We begin by introducing various stability concepts for multistep approximations of systems of ODE's which depend on a parameter. These definitions were previously stated and motivated in Section 3 of [28].

Consider the multistep method (1.9) and set $\tilde{v}^{n}=\left(v^{n}, \ldots, v^{n-r+1}\right)^{T}$, where $v^{n}$ is the solution of the corresponding homogeneous multistep method (1.8). The first definition corresponds to the usual meaning of stability in the sense of DahlquistHenrici.

Definition 3.1. (a) The multistep method (1.9) is stable if for each fixed value of $\varepsilon$ satisfying $0<\varepsilon \leqslant \varepsilon_{0}$ there exist constants $\alpha(\varepsilon), K(\varepsilon)$, and $\tau(\varepsilon)$ so that all solutions of the corresponding homogeneous multistep method (1.8) satisfy an estimate of the form

$$
\left\|\tilde{v}^{n}\right\| \leqslant K(\varepsilon) e^{\alpha(\varepsilon) t_{n-j}}\left\|\tilde{v}^{j}\right\| \text { for all } r-1 \leqslant j \leqslant n \leqslant N,
$$

when $0 \leqslant k \leqslant \tau(\varepsilon)$.

(b) The multistep method (1.9) is uniformly stable if it is stable with constants $K(\varepsilon), \alpha(\varepsilon)$, and $\tau(\varepsilon)$ which are independent of $\varepsilon$ for $0<\varepsilon \leqslant \varepsilon_{0}$.

Remark 3.2. If the matrix $A(t, \varepsilon)$ is continuous for all $0 \leqslant t \leqslant T$ and $0<\varepsilon \leqslant \varepsilon_{0}$, then method (1.9) is stable provided that the roots of the characteristic polynomial $p(\lambda)=\sum_{j=0}^{r} a_{j} \lambda^{r-j}$ satisfy the root condition. Furthermore, if $A(t, \varepsilon)$ is continuous for all $0 \leqslant t \leqslant T$ and $0 \leqslant \varepsilon \leqslant \varepsilon_{0}$, then this same algebraic condition implies that (1.9) is uniformly stable.

The next definition introduces stability concepts which are appropriate for multistep approximations of stiff systems of ODE's which depend on a parameter when the step size resolves only the slow time scales in the system of ODE's and not the fast time scales.

Definition 3.2. (a) The multistep method (1.9) is uniformly $(k, \varepsilon)$-bounded in $\Gamma$ if

$$
\begin{array}{ll}
\left(a_{0} I+b_{0} k A\left(\tau_{0}(t), \varepsilon\right)\right)^{-1} & \text { exists and is uniformly bounded for all } \\
& r k \leqslant t \leqslant T \text { and }(k, \varepsilon) \in \Gamma,
\end{array}
$$

and there exists a constant $K$ so that all solutions of the corresponding homogeneous multistep method (1.8) satisfy an estimate of the form

$$
\left\|\tilde{v}^{n}\right\| \leqslant K\left\|\tilde{v}^{n-1}\right\| \text { for all } r \leqslant n \leqslant N \text { and }(k, \varepsilon) \in \Gamma .
$$

(b) The multistep method (1.9) is uniformly $(k, \varepsilon)$-stable in $\Gamma$ if condition (3.2) is satisfied and there exist constants $K$ and $\alpha$ so that all solutions of the corresponding 
homogeneous multistep method (1.8) satisfy an estimate of the form

$$
\left\|\tilde{v}^{n}\right\| \leqslant K e^{\alpha t_{n}},\left\|\tilde{v}^{j}\right\| \text { for all } r-1 \leqslant j \leqslant n \leqslant N \text { and }(k, \varepsilon) \in \Gamma .
$$

(c) The multistep method (1.9) is strongly $(k, \varepsilon)$-stable in $\Gamma$ if condition (3.2) is satisfied and there exist constants $K$ and $0 \leqslant \delta<1$ so that all solutions of the corresponding homogeneous multistep method (1.8) satisfy an estimate of the form

$$
\left\|\tilde{v}^{n}\right\| \leqslant K \delta^{n-j}\left\|\tilde{v}^{j}\right\| \text { for all } r-1 \leqslant j \leqslant n \leqslant N \text { and }(k, \varepsilon) \in \Gamma .
$$

The following lemma states an obvious relationship among the concepts defined in Definition 3.2.

LEMMA 3.1. (a) If (1.9) is strongly $(k, \varepsilon)$-stable in $\Gamma$, then (1.9) is uniformly $(k, \varepsilon)$-stable in $\Gamma$.

(b) If (1.9) is uniformly $(k, \varepsilon)$-stable in $\Gamma$, then (1.9) is uniformly $(k, \varepsilon)$-bounded in $\Gamma$.

Remark 3.3. (1) The homogeneous multistep method (1.8) satisfies Property 1.1 if and only if there exists a trapezoidal region $\Gamma$ with the form (1.12) such that $(1.8)$ is uniformly $(k, \varepsilon)$-stable in $\Gamma$.

(2) In Theorem 2.1 we used the geometry and properties of stability domains to determine sufficient conditions under which there exists a trapezoidal region with the form (1.12) such that a multistep approximation of system (2.1) is strongly $(k, \varepsilon)$-stable in $\Gamma$.

In order to precisely state the main results which appear in Theorems 3.1,3.2, and Corollary 3.1, we find it convenient to separate implicit homogeneous multistep methods (1.8) into three different classes.

Class X: $b_{i}=0, i=1, \ldots, r$.

Class $\mathrm{Y}: \tau_{i}(t)=\tau_{0}(t)$ for $i=1, \ldots, r$, and $0 \leqslant t \leqslant T$.

Class Z: The integers $i=1, \ldots, r$, can be partitioned into two sets $I_{1}$ and $I_{2}$ such that

$$
i \in I_{1} \quad \text { if } b_{i}=0 \quad \text { or } \quad \tau_{i}(t)=\tau_{0}(t) \quad \text { for all } t \in[0, T]
$$

and

$$
i \in I_{2} \quad \text { if } b_{i} \neq 0 \quad \text { and } \quad \tau_{i}(t) \neq \tau_{0}(t) \quad \text { for all } t \in[0, T] .
$$

It is easy to check that Class $\mathrm{X}$ consists of the backward-difference methods, Class $\mathrm{Y}$ consists of the one-leg methods, and Class $\mathrm{Z}$ consists of multistep methods which are neither backward-difference methods nor one-leg methods.

Let $T(t, \varepsilon)$ denote the matrix which appears on line (1.6). For $i=1, \ldots, r$, let $E_{11}^{i}(k, t, \varepsilon), \quad E_{12}^{i}(k, t, \varepsilon), \quad E_{21}^{i}(k, t, \varepsilon), \quad E_{22}^{i}(k, t, \varepsilon) \in C^{p-1}(t, \varepsilon, B)$ denote matrices with dimensions $m \times m, m \times(n-m),(n-m) \times m$, and $(n-m) \times(n-m)$, respectively.

TheOREM 3.1. (Property 1.1 for homogeneous multistep methods (1.8).) Approximate system (1.4) by a multistep method (1.8). Assume that the multistep method is implicit, stable (i.e., its characteristic polynomial satisfies the root condition), the step size $k$ resolves the slow time scales in (1.4), that is, $k \leqslant k_{0}$, where $k_{0}$ is any constant satisfying

$$
k_{0}\left|b_{0}\right|\left\|D_{22}(t, \varepsilon)\right\|<1 \quad \text { for } 0 \leqslant t \leqslant T \text { and } 0 \leqslant \varepsilon \leqslant \varepsilon_{0},
$$


and the step size does not resolve the fast time scales in (1.4) but the fast time scales are attenuated, that is,

method (1.8) is a strongly $(k, \varepsilon)$-stable approximation of the equation $\varepsilon d w / d t=D_{11}(t, \varepsilon) w$ in $\Gamma$, where $\sigma^{*}$ is some constant and $k_{0}$ is defined by (3.8).

If (1.8) lies in Class $\mathrm{X}$ or $\mathrm{Y}$, then (1.8) is uniformly $(k, \varepsilon)$-stable in $\Gamma$.

If (1.8) lies in Class $\mathrm{Z}$ and the set $I_{2}$ is empty, then (1.8) is uniformly $(k, \varepsilon)$-stable in $\Gamma$.

If (1.8) lies in Class $\mathrm{Z}$, the set $I_{2}$ is not empty and for each $i \in I_{2}$ the matrix $T(t, \varepsilon)$ satisfies the coupling condition

$$
T^{-1}\left(\tau_{0}(t), \varepsilon\right) T\left(\tau_{i}(t), \varepsilon\right)=I+k\left(\begin{array}{ll}
E_{11}^{i}(k, t, \varepsilon) & E_{12}^{i}(k, t, \varepsilon) \\
E_{21}^{i}(k, t, \varepsilon) & E_{22}^{i}(k, t, \varepsilon)
\end{array}\right)
$$

and

$$
E_{21}^{i}(k, t, \varepsilon)=O(\varepsilon)
$$

for all $r k \leqslant t \leqslant T$ and $0 \leqslant \varepsilon \leqslant \varepsilon_{0}$, then (1.8) is uniformly $(k, \varepsilon)$-stable in $\Gamma$.

If (1.8) lies in Class Z, and there exists an integer $i \in I_{2}$ so that condition (3.10) is satisfied but condition (3.11) is violated, then (1.8) need not be uniformly $(k, \varepsilon)$-stable in $\Gamma$ for any choice of the constants $\sigma^{*}$ and $k_{0}$.

The central idea behind the proof of Theorem 3.1 is to separate the scales of the multistep approximation of (1.4). We show that after an appropriate bounded change of variables, a given multistep approximation of (1.4) can be transformed into the same multistep approximation of the system $d y / d t=D(t, \varepsilon) y$ plus a perturbation. $(D(t, \varepsilon)$ is the block diagonal matrix which appears in Assumption 1.1.) If the hypotheses in the first paragraph of Theorem 3.1 are satisfied, then we can uniformly bound the multistep approximation of $d y / d t=D(t, \varepsilon) y$ for all $(k, \varepsilon) \in \Gamma$. We use stability to bound the solution of the multistep approximation of the system $d y_{\mathrm{II}} / d t=D_{22}(t, \varepsilon) y_{\mathrm{II}}$, and we apply assumption (3.9) to bound the solution of the multistep approximation of the system $\varepsilon d y_{\mathrm{I}} / d t=D_{11}(t, \varepsilon) y_{\mathrm{I}}$. Furthermore, in the first four cases stated in Theorem 3.1, we can uniformly bound the perturbation and apply the following variant of a theorem due to Strang [39] to estimate the solution of the difference method:

LEMMA 3.2. Consider the difference approximations

$$
y^{n}=\sum_{j=1}^{r} Q_{j}\left(\tau_{0}\left(t_{n}\right), \ldots, \tau_{r}\left(t_{n}\right), k, \varepsilon\right) y^{n-j}, \quad n=r, \ldots, N,
$$

and

$$
w^{n}=\sum_{j=1}^{r} \hat{Q}_{j}\left(\tau_{0}\left(t_{n}\right), \ldots, \tau_{r}\left(t_{n}\right), k, \varepsilon\right) w^{n-j}, \quad n=r, \ldots, N
$$

where $Q_{j}, \hat{Q}_{j}, j=1, \ldots, r$, are $n \times n$ matrices. If the difference approximation (3.12) is uniformly $(k, \varepsilon)$-stable in $\Gamma$, and

$$
\hat{Q}_{j}=Q_{j}+O(k) \quad \text { for all }(k, \varepsilon) \in \Gamma \text { and } t_{r} \leqslant t_{n} \leqslant T,
$$

then the difference approximation (3.13) is also uniformly $(k, \varepsilon)$-stable in $\Gamma$. 
Proof of Theorem 3.1. The matrix $\left(a_{0} I+b_{0} k A\left(\tau_{0}(t), \varepsilon\right)\right)^{-1}$ exists and is uniformly bounded for all $r k \leqslant t \leqslant T$ and $(\hat{k}, \varepsilon) \in \Gamma$, so (1.8) can be written in the form

$$
v^{n}=\sum_{j=1}^{r} R_{j}\left(A\left(\tau_{0}\left(t_{n}\right), \varepsilon\right), A\left(\tau_{j}\left(t_{n}\right), \varepsilon\right), k, \varepsilon\right) v^{n-j}, \quad n=r, \ldots, N
$$

where

$$
R_{j}=\left(a_{0} I+k b_{0} A\left(\tau_{0}\left(t_{n}\right), \varepsilon\right)\right)^{-1}\left(a_{j} I+k b_{j} A\left(\tau_{j}\left(t_{n}\right), \varepsilon\right)\right), \quad j=1, \ldots, r .
$$

A simple application of identity (1.6) shows that

$$
\begin{aligned}
& R_{j}\left(A\left(\tau_{0}(t), \varepsilon\right), A\left(\tau_{j}(t), \varepsilon\right), k, \varepsilon\right) \\
& \quad=T\left(\tau_{0}(t), \varepsilon\right) \bar{\Lambda}_{1} T^{-1}\left(\tau_{0}(t), \varepsilon\right) T\left(\tau_{j}(t), \varepsilon\right) \bar{\Lambda}_{2} T^{-1}\left(\tau_{j}(t), \varepsilon\right)
\end{aligned}
$$

where

$$
\bar{\Lambda}_{1}=\left(\begin{array}{cc}
\left(a_{0} I+\left(\frac{b_{0} k}{\varepsilon}\right) D_{11}\left(\tau_{0}(t), \varepsilon\right)\right)^{-1} & 0 \\
0 & \left(a_{0} I+b_{0} k D_{22}\left(\tau_{0}(t), \varepsilon\right)\right)^{-1}
\end{array}\right)
$$

and

$$
\bar{\Lambda}_{2}=\left(\begin{array}{cc}
\left(a_{j} I+\left(\frac{b_{j} k}{\varepsilon}\right) D_{11}\left(\tau_{j}(t), \varepsilon\right)\right) & 0 \\
0 & \left(a_{j} I+b_{j} k D_{22}\left(\tau_{j}(t), \varepsilon\right)\right)
\end{array}\right) .
$$

Make the change of variables $v^{n}=T\left(\tau_{0}\left(t_{n}\right), \varepsilon\right) w^{n}$ in (3.15) to obtain the difference equation

$$
w^{n}=\sum_{j=1}^{r} \hat{R}_{j}\left(k, t_{n}, \varepsilon\right) w^{n-j}
$$

where

$$
\begin{aligned}
& \hat{R}_{j}(k, t, \varepsilon) \\
& \quad=T^{-1}\left(\tau_{0}(t), \varepsilon\right) R_{j}\left(A\left(\tau_{0}(t), \varepsilon\right), A\left(\tau_{j}(t), \varepsilon\right), k, \varepsilon\right) T\left(\tau_{0}(t-j k), \varepsilon\right), \\
& j=1, \ldots, r .
\end{aligned}
$$

Let $D(t, \varepsilon)$ be the matrix defined on line (1.6), and set

$$
\begin{aligned}
& R_{j}\left(D\left(\tau_{0}(t), \varepsilon\right), D\left(\tau_{j}(t), \varepsilon\right), k, \varepsilon\right) \\
& \quad=\left(a_{0} I+b_{0} k D\left(\tau_{0}(t), \varepsilon\right)\right)^{-1}\left(a_{j} I+k b_{j} D\left(\tau_{j}(t), \varepsilon\right)\right), \quad j=1, \ldots, r .
\end{aligned}
$$

If method (1.8) lies in Class X, then

$$
\begin{aligned}
& R_{j}\left(A\left(\tau_{0}(t), \varepsilon\right), A\left(\tau_{j}(t), \varepsilon\right), k, \varepsilon\right) \\
& \quad=\left(a_{0} I+b_{0} k A\left(\tau_{0}(t), \varepsilon\right)\right)^{-1}\left(a_{j} I\right), \quad j=1, \ldots, r,
\end{aligned}
$$

and

$$
\hat{R}_{j}(k, t, \varepsilon)=R_{j}\left(D\left(\tau_{0}(t), \varepsilon\right), D\left(\tau_{j}(t), \varepsilon\right), k, \varepsilon\right)+O(k)
$$
for $j=1, \ldots, r, r k \leqslant t \leqslant T$ and $(k, \varepsilon) \in \Gamma$. 
If method (1.8) lies in Class $\mathrm{Y}$, then

$$
\begin{aligned}
& R_{j}\left(A\left(\tau_{0}(t), \varepsilon\right), A\left(\tau_{j}(t), \varepsilon\right), k, \varepsilon\right) \\
& \quad=T\left(\tau_{0}(t), \varepsilon\right)\left(\begin{array}{cc}
\Lambda_{11}^{j} & 0 \\
0 & \Lambda_{22}^{j}
\end{array}\right) T^{-1}\left(\tau_{0}(t), \varepsilon\right), \quad j=1, \ldots, r,
\end{aligned}
$$

where

$$
\begin{array}{r}
\Lambda_{11}^{j}(t, \varepsilon)=\left(a_{0} I+\left(\frac{b_{0} k}{\varepsilon}\right) D_{11}\left(\tau_{0}(t), \varepsilon\right)\right)^{-1}\left(a_{j} I+\left(\frac{b_{j} k}{\varepsilon}\right) D_{11}\left(\tau_{j}(t), \varepsilon\right)\right), \\
j=1, \ldots, r,
\end{array}
$$

and

$$
\Lambda_{22}^{j}(t, \varepsilon)=\left(a_{0} I+b_{0} k D_{22}\left(\tau_{0}(t), \varepsilon\right)\right)^{-1}\left(a_{j} I+b_{j} k D_{22}\left(\tau_{j}(t), \varepsilon\right)\right), \quad j=1, \ldots, r .
$$

Consequently, $\hat{R}_{j}(k, t, \varepsilon)$ satisfies (3.19).

If method (1.8) lies in Class $\mathrm{Z}$ and the set $I_{2}$ is empty, then $\hat{R}_{j}(k, t, \varepsilon)$ satisfies (3.19).

If method (1.8) lies in Class $Z$ and $j \in I_{1}$, then $\hat{R}_{j}(k, t, \varepsilon)$ satisfies (3.19) for all $j \in I_{1}$ and $(k, \varepsilon) \in \Gamma$. If $j \in I_{2}$ and condition (3.10) is satisfied, then

$$
R_{j}\left(A\left(\tau_{0}(t), \varepsilon\right), A\left(\tau_{j}(t), \varepsilon\right), k, \varepsilon\right)=\gamma_{1}^{j}+\gamma_{2}^{j}+\gamma_{3}^{j},
$$

where

$$
\begin{gathered}
\gamma_{1}^{j}=T\left(\tau_{0}(t), \varepsilon\right)\left(\begin{array}{cc}
\Lambda_{11}^{j} & 0 \\
0 & \Lambda_{22}^{j}
\end{array}\right) T^{-1}\left(\tau_{j}(t), \varepsilon\right), \\
\gamma_{2}^{j}=k T\left(\tau_{0}(t), \varepsilon\right) \bar{\Lambda}_{1}\left(\begin{array}{cc}
E_{11}^{j}(k, t, \varepsilon) & E_{12}^{j}(k, t, \varepsilon) \\
0 & E_{22}^{j}(k, t, \varepsilon)
\end{array}\right) \bar{\Lambda}_{2} T^{-1}\left(\tau_{j}(t), \varepsilon\right),
\end{gathered}
$$

and

$$
\gamma_{3}^{j}=k T\left(\tau_{0}(t), \varepsilon\right)\left(\begin{array}{cc}
0 & 0 \\
\beta_{21}^{j} & 0
\end{array}\right) T^{-1}\left(\tau_{j}(t), \varepsilon\right)
$$

with

$$
\beta_{21}^{j}=\left(a_{0} I+k b_{0} D_{22}\left(\tau_{0}(t), \varepsilon\right)\right)^{-1} E_{21}^{j}(k, t, \varepsilon)\left(a_{j} I+\left(\frac{k b_{j}}{\varepsilon}\right) D_{11}\left(\tau_{j}(t), \varepsilon\right)\right) .
$$

$\gamma_{1}^{j}$ and $\gamma_{2}^{j}$ are uniformly bounded for all $(k, \varepsilon) \in \Gamma . \gamma_{3}^{j}$ is uniformly bounded if condition (3.11) is satisfied. Consequently, $\hat{R}_{j}(k, t, \varepsilon)$ satisfies (3.19) when (1.8) lies in Class $\mathrm{Z}$ and the coupling condition (3.10), (3.11) is satisfied for all $i \in I_{2}$.

By Remark 3.2, method (1.8) is a uniformly stable approximation of the equation $d y / d t=D_{22}(t, \varepsilon) y$ for all $0 \leqslant t \leqslant T$ and $(k, \varepsilon) \in \Gamma$. This fact and assumption (3.9) together imply that the difference equation

$$
x^{n}=\sum_{j=1}^{r} R_{j}\left(D\left(\tau_{0}(t), \varepsilon\right), D\left(\tau_{j}(t), \varepsilon\right), k, \varepsilon\right) x^{n-j}, \quad n=r, \ldots, N
$$

is uniformly $(k, \varepsilon)$-stable in $\Gamma$.

Identity (3.19) holds for the first four cases in this theorem, so we can conclude that the difference equation (3.16) is uniformly $(k, \varepsilon)$-stable in $\Gamma$ by Lemma 3.2. 
The matrices $T\left(\tau_{0}(t), \varepsilon\right)$ and $T^{-1}\left(\tau_{0}(t), \varepsilon\right)$ are uniformly bounded, so we can conclude that (3.15) is uniformly $(k, \varepsilon)$-stable in $\Gamma$ and the proof of Theorem 3.1 in the first four cases is complete. We establish the last statement in Theorem 3.1 by constructing an explicit example. This example is presented in Section 5.

ThEOREM 3.2. (Property 1.1 for inhomogeneous multistep methods (1.9).) $A p$ proximate system (1.5) by a multistep method (1.9) and set $u^{n}=\left(y^{n}, \ldots, y^{n-r+1}\right)^{T}$. Assume that method (1.9) is implicit, stable, the step size $k$ satisfies $0<k \leqslant k_{0}$ where $k_{0}$ is defined on line (3.8), and (1.9) satisfies assumption (3.9).

If the corresponding homogeneous difference method (1.8) lies in Class $\mathrm{X}$ or $\mathrm{Y}$ and $\mathscr{F}\left(\tau_{0}(t), \ldots, \tau_{r}(t), k, \varepsilon\right)=F\left(\tau_{0}(t), \varepsilon\right)$, then there exist constants $K$ and $\sigma$ such that

$$
\begin{aligned}
\left\|u^{n}\right\| \leqslant K e^{\sigma t_{n-1}}\left(\left\|u^{j}\right\|+t_{n-j} \tilde{f}\right) & \text { for all } r-1 \leqslant j \leqslant n \leqslant N \\
& \text { and }(k, \varepsilon) \in \Gamma,
\end{aligned}
$$

where

$$
\tilde{f}=\sup _{\substack{0 \leqslant t \leqslant T \\ 0 \leqslant \varepsilon \leqslant \varepsilon_{0}}}\left\|\left(f_{\mathrm{I}}(t, \varepsilon), f_{\mathrm{II}}(t, \varepsilon)\right)^{T}\right\| .
$$

That is, method (1.9) satisfies Property 1.1.

If the corresponding homogeneous difference method (1.8) lies in Class $\mathrm{Z}$, the set $\mathrm{I}_{2}$ is empty and $\mathscr{F}\left(\tau_{0}(t), \ldots, \tau_{r}(t), k, \varepsilon\right)=F\left(\tau_{0}(t), \varepsilon\right)$, then all solutions of (1.9) satisfy an estimate of the form (3.21).

If the corresponding homogeneous difference method (1.8) lies in Class $\mathrm{Z}$, the set $\mathrm{I}_{2}$ is not empty, conditions (3.10) and (3.11) are satisfied for each $i \in I_{2}$ and $\mathscr{F}\left(\tau_{0}(t), \ldots, \tau_{r}(t), k, \varepsilon\right)$ is a linear combination of $F\left(\tau_{i}(t), \varepsilon\right)$ with $i \in I_{2}$, then all solutions of (1.9) satisfy an estimate of the form (3.21).

Assume that the corresponding homogeneous difference method (1.8) lies in Class X, $\mathrm{Y}$, or Z, conditions (3.10) and (3.11) are satisfied for each $i \in I_{2}$ if (1.8) belongs to Class $\mathrm{Z}$, and that $\mathscr{F}\left(\tau_{0}(t), \ldots, \tau_{r}(t), k, \varepsilon\right)$ is a linear combination of $F\left(\tau_{i}(t), \varepsilon\right)$, $i=0, \ldots, r$. If conditions (3.10) and (3.11) are also satisfied for all $i \in I_{1}$, then all solutions of (1.9) satisfy an estimate of the form (3.21). If condition (3.10) is also satisfied for all $i \in I_{1}$, but condition (3.11) is violated for some value of $i \in I_{1}$, then the solutions of (1.9) need not satisfy an estimate of the form (3.21). Consequently, the inhomogeneous multistep method (1.9) need not satisfy Property 1.1 in this last case.

The proof of Theorem 3.2 depends on the discrete version of Duhamel's Principle and the following well-known lemma:

LEMMA 3.3. For any values of $k$ and $\varepsilon$ such that

$$
\left(a_{0} I+k b_{0} A\left(\tau_{0}(t), \varepsilon\right)\right)^{-1} \text { exists for } 0 \leqslant t \leqslant T,
$$

the multistep method (1.9) can be written as

$$
\begin{aligned}
y^{n}= & \sum_{j=1}^{r} R_{j}\left(A\left(\tau_{0}\left(t_{n}\right), \varepsilon\right), A\left(\tau_{j}\left(t_{n}\right), \varepsilon\right), k, \varepsilon\right) y^{n-j} \\
& +k \overline{\mathscr{F}}\left(\tau_{0}\left(t_{n}\right), \ldots, \tau_{r}\left(t_{n}\right), k, \varepsilon\right), \quad n=r, \ldots, N,
\end{aligned}
$$

where

$$
R_{j}=\left(a_{0} I+k b_{0} A\left(\tau_{0}\left(t_{n}\right), \varepsilon\right)\right)^{-1}\left(a_{j} I+k b_{j} A\left(\tau_{j}\left(t_{n}\right), \varepsilon\right)\right), \quad j=1, \ldots, r
$$


and

$$
\overline{\mathscr{F}}=\left(a_{0} I+k b_{0} A\left(\tau_{0}\left(t_{n}\right), \varepsilon\right)\right)^{-1} \mathscr{F}\left(\tau_{0}\left(t_{n}\right), \ldots, \tau_{r}\left(t_{n}\right), k, \varepsilon\right) .
$$

Furthermore, (3.22) can be written as the equivalent one-step method

$$
u^{n}=\alpha\left(k, t_{n}, \varepsilon\right) u^{n-1}+k G\left(k, t_{n}, \varepsilon\right), \quad n=r, \ldots, N,
$$

where

(3.26) $\alpha=\left(\begin{array}{ccc}R_{1}\left(A\left(\tau_{0}\left(t_{n}\right), \varepsilon\right), A\left(\tau_{1}\left(t_{n}\right), \varepsilon\right), k, \varepsilon\right) \cdots & R_{r}\left(A\left(\tau_{0}\left(t_{n}\right), \varepsilon\right), A\left(\tau_{r}\left(t_{n}\right), \varepsilon\right), k, \varepsilon\right) \\ I & 0 \cdots & 0 \\ 0 & I \cdots \cdots & 0 \\ 0 & 0 \cdots \cdots & 0\end{array}\right)$,

$I$ denotes the $n \times n$ identity matrix, and

$$
\begin{gathered}
u^{n}=\left(y^{n}, \ldots, y^{n-r+1}\right)^{T}, \\
\left.G\left(k, t_{n}, \varepsilon\right)=\overline{\mathscr{F}}\left(\tau_{0}\left(t_{n}\right), \ldots, \tau_{r}\left(t_{n}\right), k, \varepsilon\right), 0, \ldots, 0\right)^{T} .
\end{gathered}
$$

Proof of Theorem 3.2. We first note that in all cases listed in this theorem, method (1.9) is uniformly $(k, \varepsilon)$-stable in $\Gamma$ by Theorem 3.1 .

Method (1.9) can be written as the equivalent one-step method (3.25)-(3.27). For any integers $n$ and $j$ satisfying $r-1 \leqslant j \leqslant n \leqslant N$, the discrete version of Duhamel's Principle implies that the solution of this one-step method is given by

$$
u^{n}=S(n, j) u^{j}+k \sum_{i=j+1}^{n} S(n, i) G\left(k, t_{i}, \varepsilon\right)
$$

where

$$
S(n, j)= \begin{cases}\prod_{i=j+1}^{n} \alpha\left(k, t_{i}, \varepsilon\right) & \text { if } n \geqslant j+1, \\ I & \text { if } n=j .\end{cases}
$$

Estimate (3.28) with the triangle inequality to obtain

$$
\left\|u^{n}\right\| \leqslant\|S(n, j)\|\left\|u^{j}\right\|+k \sum_{i=j+1}^{n}\|S(n, i)\|\left\|G\left(k, t_{i}, \varepsilon\right)\right\| .
$$

In all cases listed in this theorem, (1.9) is uniformly $(k, \varepsilon)$-stable in $\Gamma$, so there exist constants $C_{1}$ and $\sigma$ so that

$$
\|S(n, j)\| \leqslant C_{1} e^{o t_{n-j}} \quad \text { for all } r-1 \leqslant j \leqslant n \leqslant N \text { and }(k, \varepsilon) \in \Gamma .
$$

Consequently, the solution of (1.9) will satisfy an estimate of the form (3.21) if there exists a constant $C_{2}$ such that

$$
\|G(k, t, \varepsilon)\| \leqslant C_{2} \tilde{f} \quad \text { for all } 0 \leqslant t \leqslant T \text { and }(k, \varepsilon) \in \Gamma .
$$

By identities (3.24) and (3.27), inequality (3.31) holds if

$$
\|\overline{\mathscr{F}}\|=\left\|\left(a_{0} I+b_{0} k A\left(\tau_{0}(t), \varepsilon\right)\right)^{-1} \mathscr{F}\left(\tau_{0}(t), \ldots, \tau_{r}(t), k, \varepsilon\right)\right\| \leqslant C_{2} \tilde{f} .
$$

Set

$$
M_{1}=\left(a_{0} I+\left(b_{0} k / \varepsilon\right) D_{11}\left(\tau_{0}(t), \varepsilon\right)\right)^{-1} \quad \text { and } \quad M_{2}=\left(a_{0} I+b_{0} k D_{22}\left(\tau_{0}(t), \varepsilon\right)\right)^{-1} \text {. }
$$


If (1.9) satisfies the conditions in any of the first three cases listed in this theorem, then

$$
\begin{aligned}
\overline{\mathscr{F}} & =\left(a_{0} I+b_{0} k A\left(\tau_{0}(t), \varepsilon\right)\right)^{-1} F\left(\tau_{0}(t), \varepsilon\right) \\
& =T\left(\tau_{0}(t), \varepsilon\right)\left(\begin{array}{cc}
M_{1} & 0 \\
0 & M_{2}
\end{array}\right) T^{-1}\left(\tau_{0}(t), \varepsilon\right) F\left(\tau_{0}(t), \varepsilon\right) \\
& =T\left(\tau_{0}(t), \varepsilon\right)\left(\begin{array}{cc}
M_{1} / \varepsilon & 0 \\
0 & M_{2}
\end{array}\right)\left(\begin{array}{l}
f_{\mathrm{I}}\left(\tau_{0}(t), \varepsilon\right) \\
f_{\mathrm{II}}\left(\tau_{0}(t), \varepsilon\right)
\end{array}\right) .
\end{aligned}
$$

The matrices $\left(M_{1} / \varepsilon\right), M_{2}$, and $T\left(\tau_{0}(t), \varepsilon\right)$ are all uniformly bounded, so we can conclude that $\overline{\mathscr{F}}$ satisfies an estimate of the form (3.32).

If (1.9) satisfies the conditions in the remaining cases listed in the theorem, then

$$
\begin{aligned}
\overline{\mathscr{F}} & =\left(a_{0} I+b_{0} k A\left(\tau_{0}(t), \varepsilon\right)\right)^{-1}\left(\sum_{i=0}^{r} \alpha_{i} F\left(\tau_{i}(t), \varepsilon\right)\right) \\
& =\sum_{i=0}^{r} \alpha_{i}\left(a_{0} I+b_{0} k A\left(\tau_{0}(t), \varepsilon\right)\right)^{-1} F\left(\tau_{i}(t), \varepsilon\right),
\end{aligned}
$$

where $\alpha_{i}, i=0, \ldots, r$, are constants. For each value of $i$,

$$
\begin{aligned}
\left(a_{0} I\right. & \left.+b_{0} k A\left(\tau_{0}(t), \varepsilon\right)\right)^{-1} F\left(\tau_{i}(t), \varepsilon\right) \\
& =T\left(\tau_{0}(t), \varepsilon\right)\left(\begin{array}{cc}
M_{1} & 0 \\
0 & M_{2}
\end{array}\right) T^{-1}\left(\tau_{0}(t), \varepsilon\right) T\left(\tau_{i}(t), \varepsilon\right) T^{-1}\left(\tau_{i}(t), \varepsilon\right) F\left(\tau_{i}(t), \varepsilon\right) \\
& =T\left(\tau_{0}(t), \varepsilon\right)\left(\begin{array}{cc}
\left(M_{1} / \varepsilon\right)\left(I+k E_{11}^{i}\right) & k M_{1} E_{12}^{i} \\
k M_{2}\left(E_{21}^{i} / \varepsilon\right) & M_{2}\left(I+k E_{22}^{i}\right)
\end{array}\right)\left(\begin{array}{l}
f_{\mathrm{I}}\left(\tau_{i}(t), \varepsilon\right) \\
f_{\mathrm{II}}\left(\tau_{i}(t), \varepsilon\right)
\end{array}\right) .
\end{aligned}
$$

All matrices which appear on the previous line are uniformly bounded if condition (3.11) is satisfied. If condition (3.11) is violated, then $k M_{2}\left(E_{21}^{i} / \varepsilon\right)=O(k / \varepsilon)$ in general, and the proof of Theorem 3.2 is complete.

Remark 3.4. (1) In [30] we construct an explicit example of a multistep method which satisfies all conditions in the last statement of Theorem 3.2 and does not satisfy Property 1.1 for a particular system of ODE's satisfying Assumptions 1.1-1.3. Consequently, the results of Theorem 3.2 are sharp.

(2) Strictly speaking, an $r$-step multistep approximation of system (1.5) satisfies Property 1.1 if:

(i) The solutions of the multistep method satisfy an estimate of the form (3.21).

(ii) The $(r-1)$ additional values of the $r$-step method, $y^{1}, \ldots, y^{r-1}$, are computed by a one-step method whose solutions satisfy an estimate of the form (3.21) for $0 \leqslant j \leqslant n \leqslant r-1$ (with $u^{j}$ replaced by $y^{j}$ ).

Consequently, the additional initial values of a multistep method must be appropriately computed in order for a multistep method to satisfy Property 1.1. This point is discussed on pp. 39-40 in [30].

In order to compare the properties of one-leg and the classical linear multistep methods, we restate Theorems 3.1 and 3.2 in terms of these methods.

Corollary 3.1. Approximate system (1.5) by a multistep method (1.9). Assume that method (1.9) is implicit, stable, and step size $k$ satisfies $k \leqslant k_{0}$, where $k_{0}$ is defined on line (3.8), and (1.9) satisfies assumption (3.9). 
If the multistep method is a one-leg method or a backward-difference method, then it is uniformly $(k, \varepsilon)$-stable in $\Gamma$ and all solutions of the inhomogeneous one-leg or backward-difference method satisfy an estimate of the form (3.21).

If the multistep method is a linear multistep method which is not a backward-difference method and the matrix $T(t, \varepsilon)$ which appears on line (1.6) satisfies the coupling condition

$$
T^{-1}(t, \varepsilon) T(t-k, \varepsilon)=I+k\left(\begin{array}{ll}
E_{11}^{1}(k, t, \varepsilon) & E_{12}^{1}(k, t, \varepsilon) \\
E_{21}^{1}(k, t, \varepsilon) & E_{22}^{1}(k, t, \varepsilon)
\end{array}\right)
$$

and

$$
E_{21}^{1}=O(\varepsilon)
$$

for all $0 \leqslant t \leqslant T$ and $0 \leqslant \varepsilon \leqslant \varepsilon_{0}$, where $E_{i j}^{1}, i, j=1,2$, are characterized in Theorem 3.1 , then the multistep method is uniformly $(k, \varepsilon)$-stable in $\Gamma$. Furthermore, all solutions of the inhomogeneous linear multistep method satisfy an estimate of the form (3.21).

If the multistep method is a linear multistep method which is not a backward-difference method and condition (3.33) is satisfied but condition (3.34) is violated, then the linear multistep method need not be uniformly $(k, \varepsilon)$-stable in $\Gamma$ for any choice of the constants $\sigma^{*}$ and $k_{0}$.

We now discuss the coupling condition (3.33), (3.34) before making some remarks about the practical implications of Corollary 3.1.

Assume that the matrix $T(t, \varepsilon)$ which appears on line (1.6) satisfies $T(t, \varepsilon) \in$ $C^{2}(t, \varepsilon, B)$. Make the change of variables $y=T(t, \varepsilon) w$ in system (1.5) to obtain the equation

$$
\frac{d w}{d t}=\left(D(t, \varepsilon)-T^{-1}(t, \varepsilon) \frac{d T(t, \varepsilon)}{d t}\right) w+T^{-1}(t, \varepsilon) F(t, \varepsilon),
$$

where the matrix $D(t, \varepsilon)$ is defined on line (1.6). Identity (3.35) shows that the matrix $-\left(T^{-1}(t, \varepsilon) d T(t, \varepsilon) / d t\right)$ controls the coupling between the fast and slow time scales in system (1.5).

Now consider the expression on line (3.33). By Taylor's Theorem

$$
T(t-k, \varepsilon)=T(t, \varepsilon)-k \frac{d T(t, \varepsilon)}{d t}+O\left(k^{2}\right)
$$

for $0 \leqslant t \leqslant T$ and $0 \leqslant \varepsilon \leqslant \varepsilon_{0}$.

Substitute (3.36) into (3.33) and let $k \rightarrow 0$ to obtain

$$
-T^{-1}(t, \varepsilon) \frac{d T(t, \varepsilon)}{d t}=\left(\begin{array}{ll}
E_{11}^{1}(0, t, \varepsilon) & E_{12}^{1}(0, t, \varepsilon) \\
E_{21}^{1}(0, t, \varepsilon) & E_{22}^{1}(0, t, \varepsilon)
\end{array}\right) .
$$

If condition (3.34) is also satisfied, then

$$
-T^{-1}(t, \varepsilon) \frac{d T(t, \varepsilon)}{d t}=\left(\begin{array}{cc}
E_{11}^{1}(0, t, \varepsilon) & E_{12}^{1}(0, t, \varepsilon) \\
0 & E_{22}^{1}(0, t, \varepsilon)
\end{array}\right)+O(\varepsilon) .
$$

Identity (3.38) shows that conditions (3.33) and (3.34) impose a restriction on the strength of the coupling from the fast to the slow time scales of system (1.5). In particular, conditions (3.33) and (3.34) are satisfied if the coupling from the fast time 
scales of (1.5) to the slow time scales of (1.5) is sufficiently weak. For this reason, conditions (3.33) and (3.34) are called the coupling condition in this paper and in [28]. A detailed discussion of the coupling condition can be found in van Veldhuizen [42].

Condition (3.38) clearly places a restriction on the structure of system (1.4) and the matrix $T(t, \varepsilon)$ in particular. If (3.38) is satisfied, then standard results from the theory of ODE's imply that

$$
\begin{aligned}
T(t, \varepsilon)=T(0, \varepsilon)\left(\begin{array}{cc}
M_{11}(t, \varepsilon) & M_{12}(t, \varepsilon) \\
0 & M_{22}(t, \varepsilon)
\end{array}\right)+ & O(\varepsilon) \\
& \text { for } 0 \leqslant t \leqslant T \text { and } 0 \leqslant \varepsilon \leqslant \varepsilon_{0},
\end{aligned}
$$

where $M_{11}$ is an $m \times m$ matrix, $M_{12}$ is an $m \times(n-m)$ matrix, and $M_{22}$ is an $(n-m) \times(n-m)$ matrix. We obtain the following result:

LEMMA 3.4. If

(1) the matrix $A(t, \varepsilon)$ in (1.4) is constant, or

(2) system (1.4) appears in singular perturbation form and has no turning points, that is,

$$
A(t, \varepsilon)=\left(\begin{array}{cc}
\frac{A_{11}(t, \varepsilon)}{\varepsilon} & \frac{A_{12}(t, \varepsilon)}{\varepsilon} \\
A_{21}(t, \varepsilon) & A_{22}(t, \varepsilon)
\end{array}\right)
$$

where $A_{i j}(t, \varepsilon) \in C^{p}(t, \varepsilon, B)$ for $i, j=1,2$, and $A_{11}(t, \varepsilon)$ is invertible with $A_{11}^{-1}(t, \varepsilon) \in$ $C^{p}(t, \varepsilon, B)$, then condition (3.39) is always satisfied.

The proof of part (1) of Lemma 3.4 is obvious and a proof of part (2) can be found in Section 2 of [29].

It is well known that a one-leg method and its corresponding linear multistep method have identical properties when they approximate systems of ODE's with constant coefficients. Furthermore, there is an invertible transformation which transforms the solution of a linear multistep method into its corresponding one-leg twin (see Dahlquist [8]). Despite these facts, Dahlquist and Lindberg [11], Dahlquist [10], Nevanlinna and Liniger [33] and van Veldhuizen [42] have demonstrated that in various ways the behavior of a linear multistep method and its corresponding one-leg method can be very different when they approximate variable coefficient (and nonlinear) stiff problems. We make the following additional observations about these two classes of methods on the basis of Corollary 3.1:

1. The uniform $(k, \varepsilon)$-stability (stability with large step sizes) of linear multistep approximations of stiff linear systems depends on the form in which system (1.5) appears. This comment is not true for one-leg methods. Consider the cases when the matrix $A(t, \varepsilon)$ in (1.5) is constant or system (1.5) appears in singular perturbation form without turning points, that is, system (1.5) has the form

$$
\begin{aligned}
\varepsilon \frac{d y_{\mathrm{I}}}{d t} & =A_{11}(t, \varepsilon) y_{\mathrm{I}}+A_{12}(t, \varepsilon) y_{\mathrm{II}}+f_{\mathrm{I}}(t, \varepsilon), \\
\frac{d y_{\mathrm{II}}}{d t} & =A_{21}(t, \varepsilon) y_{\mathrm{I}}+A_{22}(t, \varepsilon) y_{\mathrm{II}}+f_{\mathrm{II}}(t, \varepsilon),
\end{aligned}
$$


where the matrices $A_{i j}(t, \varepsilon), i=1,2, j=1,2$, are characterized in Lemma 3.4 and $f_{\mathrm{I}}(t, \varepsilon), f_{\mathrm{II}}(t, \varepsilon) \in C^{p}(t, \varepsilon, B)$. In these two cases, the uniform $(k, \varepsilon)$-stability of both a one-leg approximation of system (1.5) and its corresponding linear multistep approximation are determined by the usual Dahlquist-Henrici stability theory to control the the slow time scales of (1.5), and by the use of stability domains to control the fast time scales. If system (1.5) satisfies the conditions in Assumptions $1.1-1.3$ but is otherwise unrestricted, then the two previously mentioned properties determine the uniform $(k, \varepsilon)$-stability of a one-leg approximation of (1.5). The uniform $(k, \varepsilon)$-stability of a linear multistep approximation of system (1.5) depends on an additional condition, namely, the coupling condition (3.33), (3.34). This condition only arises when the coefficients in system (1.5) vary with time.

We note that if we make the change of variables $y(t)=T(t, \varepsilon) w$ in (1.5), then the resulting equation for $w$ appears in singular perturbation form. According to Corollary 3.1 , the uniform $(k, \varepsilon)$-stability properties of a linear multistep approximation of the scaled system which $w$ satisfies are better than the uniform $(k, \varepsilon)$-stability properties of the linear multistep approximation of the original system (1.5).

2. If we consider problems which exhibit a strong coupling from the fast time scales to the slow time scales, then a one-leg method using a given step size $k$ can compute an accurate solution of the equation, while the solution of its corresponding linear multistep method using the same step size will produce a solution which is not accurate at all. In other words, the linear multistep method requires a smaller step size to obtain the same accuracy as the corresponding one-leg method. This point is illustrated with an example in Section 5.

Remark 2 is consistent with observations made in [14] and [15], which demonstrate that the integration package TRAPEX (based on the trapezoidal rule with extrapolation) has difficulties solving nonlinear systems of ODE's when there is a strong coupling between the slow and fast time scales. According to Corollary 3.1, these numerical difficulties could be avoided if the trapezoidal rule is replaced by the implicit midpoint rule. This change was made by Lindberg [26], [27] in the integration package IMPEX.

3. Corollary 3.1 demonstrates that one-leg methods have better stability properties with large step sizes, in general, than their linear multistep counterparts. Consequently, one-leg methods are to be preferred over their linear multistep counterparts when choosing a discretization for a system of stiff ODE's.

4. The Relationship Between Property 1.1 and $D$-Stability. In this section we relate the theory developed in Section 3 to the concept of $D$-stability introduced by van Veldhuizen in [42]. In [42] van Veldhuizen stated that $D$-stability complements the usual notions of stability and stability domains, and that $D$-stability has no relation to the propagation of errors for solutions of difference methods. An important consequence of the results in this section is that we relate the concept of $D$-stability to the usual notions of stability and stability domains, and to the propagation of errors for multistep methods which use large step sizes.

Following van Veldhuizen [42], consider the homogeneous model problem

$$
d y / d t=A(t) y, \quad t \geqslant t_{0},
$$


where $A(t)$ is a matrix. Approximate system (4.1) by a discretization method which can be written in the form

$$
y_{i+1}=G\left(t_{i}, k\right) y_{i}
$$

where $k$ is the step size, $t_{i}=t_{0}+i k$, and $G\left(t_{i}, k\right)$ is a matrix. If the discretization is a one-step method, then $y_{i}$ is an approximation to $y\left(t_{i}\right)$. If the discretization is a multistep method, then the recursion (4.2) describes the canonical one-step recursion corresponding to the multistep method.

Let $T, k_{0}$, and $\lambda$ denote given constants.

Definition 4.1 (van Veldhuizen). The discretization of (4.1) resulting in the recursion (4.2) is called $D(\mathscr{L})$-stable if for all stiff systems in the Class $\mathscr{L}$, for all $t \in\left[t_{0}, t_{0}+T\right]$ and all $k \in\left(0, k_{0}\right]$

$$
\|G(t, k)\| \leqslant M<\infty,
$$

where $M$ is a constant depending only on $k_{0}$ and the Class $\mathscr{L}$.

Van Veldhuizen makes the following choice for the Class $\mathscr{L}$ :

Definition 4.2 (Class $\mathscr{L}$ ). The Class $\mathscr{L}$ of stiff systems consists of all linear systems (4.1) parametrized by a parameter $\varepsilon \in\left(0, \varepsilon_{0}\right]$ which satisfy the following conditions:

(S1) $y(t) \in \mathbf{C}^{2}$

(S2) $A(t)=T(t) \Lambda T^{-1}(t)$ for all $t \in\left[t_{0}, t_{0}+T\right]$. Here $\Lambda(t)$ is a diagonal matrix given by

$$
\Lambda(t)=\left(\begin{array}{cc}
\lambda(t) / \varepsilon & 0 \\
0 & \mu(t)
\end{array}\right)
$$

where $\operatorname{Re}(\lambda(t)) \leqslant \lambda<0$ for all $t \in\left[t_{0}, t_{0}+T\right]$.

(S3) $\lambda, \mu, T, T^{-1}$ depend smoothly on $t$ and possibly $\varepsilon \in\left(0, \varepsilon_{0}\right]$, and the derivatives from order zero up to a certain order (sufficiently high) are bounded uniformly in $t$ and $\varepsilon \in\left(0, \varepsilon_{0}\right]$.

Discretizations of systems (4.1) fall into two categories. Either all time scales in (4.1) are resolved by the step size or some time scales in (4.1) are not resolved by the step size. (For systems which lie in Class $\mathscr{L}$, all scales are resolved if $k / \varepsilon \rightarrow 0$ as $k \rightarrow 0$. The fast time scales are not resolved if $k / \varepsilon \geqslant \sigma^{*}$ with $\sigma^{*}$ a constant as $k \rightarrow 0$.) If all scales in (4.1) are resolved by the step size, then the usual DahlquistHenrici stability theory controls the error propagation properties of the multistep discretization. A meaningful stability concept for a multistep discretization of stiff equations which complements the Dahlquist-Henrici theory should apply to the case where some time scales in (4.1) are not resolved by the step size. Consequently, we alter van Veldhuizen's Definition 4.1 for systems (4.1) which depend on a parameter $0<\varepsilon<\varepsilon_{0}$ to reflect the complementary case when all scales in (4.1) are not resolved by the step size.

Let $\Gamma$ denote the trapezoidal region defined on line (1.12).

Definition 4.1a. Consider systems (1.4). The discretization of (1.4) resulting in the recursion

$$
y_{i+1}=G\left(t_{i}, k, \varepsilon\right) y_{i}
$$


is called $\bar{D}(\mathscr{L})$-stable if for all stiff systems in the Class $\mathscr{L}$, for all $t \in\left[t_{0}, t_{0}+T\right]$ and $(k, \varepsilon) \in \Gamma$,

$$
\|G(t, k, \varepsilon)\| \leqslant M<\infty
$$

where $M$ is a constant depending only on $k_{0}, \sigma^{*}$, and the Class $\mathscr{L}$.

We also replace the Class $\mathscr{L}$ defined in Definition 4.2 by the Class $C$ which consists of all systems (1.4) (or a subset of systems (1.4)) which satisfy the conditions in Assumptions 1.1 and 1.2.

We are now prepared to state two results which connect our version of van Veldhuizen's Definition 4.1 to the results in Section 3 and to the propagation of errors for multistep methods which use large step sizes.

THEOREM 4.1. Approximate a system in Class $C$ by a multistep method (1.8). The multistep method is $\bar{D}(C)$-stable if and only if for all stiff systems in $C$, the multistep method is uniformly $(k, \varepsilon)$-bounded in $\Gamma$ with a bound which only depends on the class C.

THEOREM 4.2. If the hypotheses in Theorem 3.1 hold uniformly for all systems in Class $C$, then the multistep method is $\bar{D}(C)$-stable. Furthermore, for all systems in $C$ the multistep method is uniformly $(k, \varepsilon)$-stable in $\Gamma$ with constants $K$ and $\sigma$ (which appear on line (3.4)) depending only on the Class $C$.

We have applied Part (b) of Lemma 3.1 to justify the first statement in Theorem 4.2.

5. Numerical Examples and the Practical Implications of Property 1.1. In this section, we introduce a model variable coefficient system of ODE's which satisfies the conditions in Assumptions 1.1-1.3. We approximate this model equation by a particular one-leg method and its corresponding linear multistep method. Our goal is to illustrate the practical consequences of Corollary 3.1 which are stated at the end of Section 3, and to make a general comparison between the classes of one-leg and linear multistep methods.

Consider the model system

$$
d v / d t=\left\{U^{*}(t) D U(t)\right\} v, \quad 0 \leqslant t<\infty, 0<\varepsilon \leqslant .1, v(0)=(1.0,1.0)^{T},
$$

where

$$
U(t)=\left(\begin{array}{cc}
\cos (t) & \sin (t) \\
-\sin (t) & \cos (t)
\end{array}\right), \quad D=\left(\begin{array}{cc}
-1 / \varepsilon & 0 \\
0 & -1
\end{array}\right)
$$

and $U^{*}(t)$ denotes the transpose of $U(t)$.

System (5.1) satisfies the conditions in Assumptions 1.1 and 1.2 with the matrices $T(t, \varepsilon)$ and $D(t, \varepsilon)$ which appear in Assumption 1.1 given by

$$
T(t, \varepsilon)=U^{*}(t) \text { and } D(t, \varepsilon)=D .
$$

The matrix $U(t)$ defined on line (5.2) is unitary, so

$$
\begin{aligned}
T^{-1}(t, \varepsilon) T(t-k, \varepsilon) & =U(t) U^{*}(t-k)=\left(\begin{array}{cc}
\cos (k) & \sin (k) \\
-\sin (k) & \cos (k)
\end{array}\right) \\
& =I+k\left(\begin{array}{cc}
0 & 1 \\
-1 & 0
\end{array}\right)+O\left(k^{2}\right) .
\end{aligned}
$$


Consequently, system (5.1) does not satisfy the coupling condition (3.33), (3.34).

System (5.1) can be transformed into a system of ODE's with constant coefficients. Make the change of variables $v=U^{*}(t) w$ in (5.1) to obtain

$$
\frac{d w}{d t}=\left(\begin{array}{cc}
-1 / \varepsilon & -1 \\
1 & -1
\end{array}\right) w, \quad w(0)=v(0) .
$$

Equation (5.4) is easily solved to produce the general solution of (5.1) which is given by

$$
v(t)=U^{*}(t) M\left(\begin{array}{cc}
e^{\lambda_{-} t} & 0 \\
0 & e^{\lambda_{+} t}
\end{array}\right) M^{-1} v(0)
$$

where

$$
\begin{gathered}
M=\left(\begin{array}{cc}
1 & \frac{2 \varepsilon}{1+J-\varepsilon} \\
\frac{2 \varepsilon}{1+J-\varepsilon} & 1
\end{array}\right), \\
\lambda_{ \pm}=\frac{1}{2}\left(-\left(1+\frac{1}{\varepsilon}\right) \pm \frac{1}{\varepsilon} J\right), \quad \text { and } J=\sqrt{1-2 \varepsilon-3 \varepsilon^{2}} .
\end{gathered}
$$

A simple calculation shows that

$$
\lambda_{-}=-1 / \varepsilon+O(1) \quad \text { and } \quad \lambda_{+}=-1+O(\varepsilon) \quad \text { as } \varepsilon \rightarrow 0 .
$$

We conclude that the solutions of (5.1) behave like the solutions of the constant coefficient system $d \tilde{v} / d t=D \tilde{v}$. Furthermore, any solution of (5.1) can be written as the sum of a slowly varying solution of $(5.1)$, denoted by $v^{s}(t)$, and a rapidly varying solution of (5.1), denoted by $v^{R}(t)$, namely,

$$
v(t)=v^{s}(t)+v^{R}(t),
$$

where

$$
v^{s}(t)=U^{*}(t) M\left(\begin{array}{cc}
0 & 0 \\
0 & e^{\lambda_{+} t}
\end{array}\right) M^{-1} v(0)
$$

and

$$
v^{R}(t)=U^{*}(t) M\left(\begin{array}{cc}
e^{\lambda_{-} t} & 0 \\
0 & 0
\end{array}\right) M^{-1} v(0) .
$$

Consider the one-leg approximation of (5.1) given by

$$
\begin{aligned}
\left(I-k U^{*}\right. & \left.\left(\frac{3}{4} t_{n+1}+\frac{1}{4} t_{n}\right) D U\left(\frac{3}{4} t_{n+1}+\frac{1}{4} t_{n}\right)\right) x^{n+1} \\
& =\left(I+k U^{*}\left(\frac{3}{4} t_{n+1}+\frac{1}{4} t_{n}\right) D U\left(\frac{3}{4} t_{n+1}+\frac{1}{4} t_{n}\right)\right) x^{n}, \quad x^{0}=v(0) .
\end{aligned}
$$

The corresponding one-step approximation of (5.1) is given by

$$
\begin{aligned}
\left(I-\frac{3 k}{4}\right. & \left.U^{*}\left(t_{n+1}\right) D U\left(t_{n+1}\right)\right) y^{n+1} \\
& =\left(I+\frac{k}{4} U^{*}\left(t_{n}\right) D U\left(t_{n}\right)\right) y^{n}, \quad y^{0}=v(0) .
\end{aligned}
$$

Let $\Gamma$ denote the trapezoidal region

$$
\Gamma=\left\{(k, \varepsilon) \in \mathbf{R}^{2}: 0<\varepsilon \leqslant .1 \text { and } \sigma^{*} \varepsilon \leqslant k \leqslant k_{0}\right\},
$$


where $\sigma^{*}$ is any positive constant and $k_{0}$ is any constant satisfying $k_{0}<4 / 3$. Both the one-leg method (5.5) and its corresponding one-step method (5.6) are implicit, stable, and strongly $(k, \varepsilon)$-stable approximations of $\varepsilon \dot{y}=-y$ in $\Gamma$. Corollary 3.1 implies that $(5.5)$ is uniformly $(k, \varepsilon)$-stable in $\Gamma$.

We now show that method (5.6) is not a uniformly $(k, \varepsilon)$-stable approximation of system (5.1) in $\Gamma$ for any choice of the constant $\sigma^{*}$. Since method (5.6) satisfies all hypotheses in the first paragraph of Corollary 3.1, but system (5.1) does not satisfy the coupling condition (3.33), (3.34), we conclude that the results in Corollary 3.1 (and Theorem 3.1) are sharp.

The matrix

$$
\left(I-\frac{3 k}{4} U^{*}(t) D U(t)\right)
$$

is invertible for all $t \geqslant 0$ and $(k, \varepsilon) \in \Gamma$, so method (5.6) can be written as

$$
\begin{aligned}
y^{n+1} & =\left(I-\frac{3 k}{4} U^{*}\left(t_{n+1}\right) D U\left(t_{n+1}\right)\right)^{-1}\left(I+\frac{k}{4} U^{*}\left(t_{n}\right) D U\left(t_{n}\right)\right) y^{n} \\
& =\left(U^{*}\left(t_{n+1}\right)\left(I-\frac{3 k}{4} D\right)^{-1} U\left(t_{n+1}\right) U^{*}\left(t_{n}\right)\left(I+\frac{k}{4} D\right) U\left(t_{n}\right)\right) y^{n} \\
& =\left(\begin{array}{ll}
M_{11} & M_{12} \\
M_{21} & M_{22}
\end{array}\right) y^{n}
\end{aligned}
$$

where $M_{11}, M_{12}$, and $M_{22}$ are uniformly bounded in $\Gamma$ and

$$
\begin{aligned}
M_{21}= & \left(1+\frac{3 k}{4}\right)^{-1}\left(1-\frac{k}{4 \varepsilon}\right) \cos \left(t_{n+1}\right) \cos \left(t_{n}\right) \\
& \times\left(\sin \left(t_{n}\right) \cos \left(t_{n+1}\right)-\sin \left(t_{n+1}\right) \cos \left(t_{n}\right)\right)+E,
\end{aligned}
$$

where $E$ is uniformly bounded in $\Gamma$. A simple calculation using Taylor's Theorem shows that

$$
M_{21}=-\frac{k^{2}}{4 \varepsilon} \cos ^{2}\left(t_{n}\right)+O\left(\frac{k^{3}}{\varepsilon}\right) \quad \text { for all }(k, \varepsilon) \in \Gamma .
$$

Clearly, $M_{21}$ is not uniformly bounded in $\Gamma$ for any choice of the constants $\sigma^{*}>0$ and $k_{0}$ as $\varepsilon \rightarrow 0$. Consequently, the one-step method (5.6) is not uniformly $(k, \varepsilon)$ bounded in $\Gamma$. By part (b) of Lemma 3.1 we conclude that the one-step method (5.6) is not uniformly $(k, \varepsilon)$-stable in $\Gamma$ for any choice of the constants $\sigma^{*}$ and $k_{0}$.

We now examine some numerical results which illustrate the practical importance of uniform $(k, \varepsilon)$-stability. Let $v\left(t_{n}\right)=\left(v_{\mathrm{I}}\left(t_{n}\right), v_{\mathrm{II}}\left(t_{n}\right)\right)^{T}$ and $v^{s}\left(t_{n}\right)=$ $\left(v_{\mathrm{I}}^{s}\left(t_{n}\right), v_{\mathrm{II}}^{s}\left(t_{n}\right)\right)^{T}$ denote the exact solution of (5.1) and the slowly varying part of $v\left(t_{n}\right)$, respectively, at the grid points $t_{n}=n k, n=0,1, \ldots$, with $k=.1$. Let $x^{n}=\left(x_{\mathrm{I}}^{n}, x_{\mathrm{II}}^{n}\right)^{T}$ and $y^{n}=\left(y_{\mathrm{I}}^{n}, y_{\mathrm{II}}^{n}\right)^{T}$ denote the solutions of (5.5) and (5.6), respectively, at the grid points $t_{n}=n k . v\left(t_{n}\right), v^{s}\left(t_{n}\right), x^{n}$, and $y^{n}$ were computed in double precision on the IBM 3081 at Brown University. Values of these quantities at selected grid points are listed in the following tables for the three cases $\varepsilon=2.5 \times$ $10^{-3}, \varepsilon=2.5 \times 10^{-6}$, and $\varepsilon=2.5 \times 10^{-9}$. 
$\varepsilon=2.5 \times 10^{-3}$

\begin{tabular}{rrrrr}
\multicolumn{1}{c}{$n$} & \multicolumn{1}{c}{$v_{\mathrm{I}}\left(t_{n}\right)$} & \multicolumn{1}{c}{$v_{\mathrm{I}}^{s}\left(t_{n}\right)$} & \multicolumn{1}{c}{$x_{\mathrm{I}}^{n}$} & \multicolumn{1}{c}{$y_{\mathrm{I}}^{n}$} \\
\hline 0 & $.100 \mathrm{D}+01$ & $.251 \mathrm{D}-02$ & $.100 \mathrm{D}+01$ & $.100 \mathrm{D}+01$ \\
1 & $-.881 \mathrm{D}-01$ & $-.881 \mathrm{D}-01$ & $-.374 \mathrm{D}+00$ & $-.459 \mathrm{D}+00$ \\
10 & $-.308 \mathrm{D}+00$ & $-.308 \mathrm{D}+00$ & $-.294 \mathrm{D}+00$ & $-.525 \mathrm{D}+00$ \\
50 & $.639 \mathrm{D}-02$ & $.639 \mathrm{D}-02$ & $.608 \mathrm{D}-02$ & $.109 \mathrm{D}-01$ \\
75 & $-.509 \mathrm{D}-03$ & $-.509 \mathrm{D}-03$ & $-.486 \mathrm{D}-03$ & $-.868 \mathrm{D}-03$ \\
100 & $.240 \mathrm{D}-04$ & $.240 \mathrm{D}-04$ & $.230 \mathrm{D}-04$ & $.410 \mathrm{D}-04$ \\
114 & $.100 \mathrm{D}-04$ & $.100 \mathrm{D}-04$ & $.956 \mathrm{D}-05$ & $.171 \mathrm{D}-04$
\end{tabular}

\begin{tabular}{rcccc}
\multicolumn{1}{c}{$n$} & $v_{\mathrm{II}}\left(t_{n}\right)$ & $v_{\mathrm{II}}^{s}\left(t_{n}\right)$ & $x_{\mathrm{II}}^{n}$ & $y_{\mathrm{II}}^{n}$ \\
\hline 0 & $.100 \mathrm{D}+01$ & $.100 \mathrm{D}+01$ & $.100 \mathrm{D}+01$ & $.100 \mathrm{D}+01$ \\
1 & $.900 \mathrm{D}+00$ & $.900 \mathrm{D}+00$ & $.813 \mathrm{D}+00$ & $.171 \mathrm{D}+01$ \\
10 & $.199 \mathrm{D}+00$ & $.199 \mathrm{D}+00$ & $.189 \mathrm{D}+00$ & $.339 \mathrm{D}+00$ \\
50 & $.187 \mathrm{D}-02$ & $.187 \mathrm{D}-02$ & $.180 \mathrm{D}-02$ & $.319 \mathrm{D}-02$ \\
75 & $.189 \mathrm{D}-03$ & $.189 \mathrm{D}-03$ & $.180 \mathrm{D}-03$ & $.323 \mathrm{D}-03$ \\
100 & $-.372 \mathrm{D}-04$ & $-.372 \mathrm{D}-04$ & $-.360 \mathrm{D}-04$ & $-.636 \mathrm{D}-04$ \\
114 & $.426 \mathrm{D}-05$ & $.426 \mathrm{D}-05$ & $.408 \mathrm{D}-05$ & $.727 \mathrm{D}-05$
\end{tabular}

$\varepsilon=2.5 \times 10^{-6}$

\begin{tabular}{rrrrr}
\multicolumn{1}{c}{$n$} & \multicolumn{1}{c}{$v_{\mathrm{I}}\left(t_{n}\right)$} & \multicolumn{1}{c}{$v_{\mathrm{I}}^{s}\left(t_{n}\right)$} & \multicolumn{1}{c}{$x_{\mathrm{I}}^{n}$} & \multicolumn{1}{c}{$y_{\mathrm{I}}^{n}$} \\
\hline 0 & $.100 \mathrm{D}+01$ & $.250 \mathrm{D}-05$ & $.100 \mathrm{D}+01$ & $.100 \mathrm{D}+01$ \\
1 & $-.903 \mathrm{D}-01$ & $-.903 \mathrm{D}-01$ & $-.419 \mathrm{D}+00$ & $-.931 \mathrm{D}+02$ \\
10 & $-.310 \mathrm{D}+00$ & $-.310 \mathrm{D}+00$ & $-.295 \mathrm{D}+00$ & $-.232 \mathrm{D}+03$ \\
50 & $.646 \mathrm{D}-02$ & $.646 \mathrm{D}-02$ & $.616 \mathrm{D}-02$ & $.486 \mathrm{D}+01$ \\
75 & $-.519 \mathrm{D}-03$ & $-.519 \mathrm{D}-03$ & $-.496 \mathrm{D}-03$ & $-.391 \mathrm{D}+00$ \\
100 & $.247 \mathrm{D}-04$ & $.247 \mathrm{D}-04$ & $.237 \mathrm{D}-04$ & $.186 \mathrm{D}-01$ \\
114 & $.103 \mathrm{D}-04$ & $.103 \mathrm{D}-04$ & $.984 \mathrm{D}-05$ & $.777 \mathrm{D}-02$
\end{tabular}

\begin{tabular}{rcccc}
\multicolumn{1}{c}{$n$} & $v_{\mathrm{II}}\left(t_{n}\right)$ & $v_{\mathrm{II}}^{s}\left(t_{n}\right)$ & $x_{\mathrm{II}}^{n}$ & $y_{\mathrm{II}}^{n}$ \\
\hline 0 & $.100 \mathrm{D}+01$ & $.100 \mathrm{D}+01$ & $.100 \mathrm{D}+01$ & $.100 \mathrm{D}+01$ \\
1 & $.900 \mathrm{D}+00$ & $.900 \mathrm{D}+00$ & $.807 \mathrm{D}+00$ & $.925 \mathrm{D}+03$ \\
10 & $.199 \mathrm{D}+00$ & $.199 \mathrm{D}+00$ & $.189 \mathrm{D}+00$ & $.149 \mathrm{D}+03$ \\
50 & $.191 \mathrm{D}-02$ & $.191 \mathrm{D}-02$ & $.184 \mathrm{D}-02$ & $.144 \mathrm{D}+01$ \\
75 & $.192 \mathrm{D}-03$ & $.192 \mathrm{D}-03$ & $.182 \mathrm{D}-03$ & $.144 \mathrm{D}+00$ \\
100 & $-.381 \mathrm{D}-04$ & $-.381 \mathrm{D}-04$ & $-.364 \mathrm{D}-04$ & $-.287 \mathrm{D}-01$ \\
114 & $.441 \mathrm{D}-05$ & $.441 \mathrm{D}-05$ & $.424 \mathrm{D}-05$ & $.332 \mathrm{D}-02$
\end{tabular}




\begin{tabular}{rrrrr}
\multicolumn{1}{c}{$\varepsilon=2.5 \times 10^{-9}$} & & & \\
\multicolumn{1}{c}{$n$} & $v_{\mathrm{I}}\left(t_{n}\right)$ & $v_{\mathrm{I}}^{s}\left(t_{n}\right)$ & $x_{\mathrm{I}}^{n}$ & \multicolumn{1}{c}{$y_{\mathrm{I}}^{n}$} \\
\hline 0 & $.100 \mathrm{D}+01$ & $.250 \mathrm{D}-08$ & $.100 \mathrm{D}+01$ & $.100 \mathrm{D}+01$ \\
1 & $-.903 \mathrm{D}-01$ & $-.903 \mathrm{D}-01$ & $-.419 \mathrm{D}+00$ & $-.927 \mathrm{D}+05$ \\
10 & $-.310 \mathrm{D}+00$ & $-.310 \mathrm{D}+00$ & $-.295 \mathrm{D}+00$ & $-.232 \mathrm{D}+06$ \\
50 & $.646 \mathrm{D}-02$ & $.646 \mathrm{D}-02$ & $.616 \mathrm{D}-02$ & $.486 \mathrm{D}+04$ \\
75 & $-.519 \mathrm{D}-03$ & $-.519 \mathrm{D}-03$ & $-.496 \mathrm{D}-03$ & $-.390 \mathrm{D}+03$ \\
100 & $.247 \mathrm{D}-04$ & $.247 \mathrm{D}-04$ & $.237 \mathrm{D}-04$ & $.186 \mathrm{D}+02$ \\
114 & $.103 \mathrm{D}-04$ & $.103 \mathrm{D}-04$ & $.984 \mathrm{D}-05$ & $.776 \mathrm{D}+01$ \\
& & & & $y_{\mathrm{II}}^{n}$ \\
$n$ & $v_{\mathrm{II}}\left(t_{n}\right)$ & $v_{\mathrm{II}}^{s}\left(t_{n}\right)$ & .100 & $.100 \mathrm{D}+01$ \\
\hline 0 & $.100 \mathrm{D}+01$ & $.100 \mathrm{D}+01$ & $.100 \mathrm{D}+01$ & $.924 \mathrm{D}+06$ \\
1 & $.900 \mathrm{D}+00$ & $.900 \mathrm{D}+00$ & $.807 \mathrm{D}+00$ & $.149 \mathrm{D}+06$ \\
10 & $.199 \mathrm{D}+00$ & $.199 \mathrm{D}+00$ & $.189 \mathrm{D}+00$ & $.144 \mathrm{D}+04$ \\
50 & $.191 \mathrm{D}-02$ & $.191 \mathrm{D}-02$ & $.184 \mathrm{D}-02$ & $.144 \mathrm{D}+03$ \\
75 & $.192 \mathrm{D}-03$ & $.192 \mathrm{D}-03$ & $.182 \mathrm{D}-03$ & $-.287 \mathrm{D}+02$ \\
100 & $-.381 \mathrm{D}-04$ & $-.381 \mathrm{D}-04$ & $-.364 \mathrm{D}-04$ & $.332 \mathrm{D}+01$ \\
114 & $.441 \mathrm{D}-05$ & $.441 \mathrm{D}-05$ & $.424 \mathrm{D}-05$ &
\end{tabular}

Remark 5.1. We list both the exact solution of (5.1) and the slowly varying part of the solution of (5.1) in order to illustrate Property 1.2 .

These numerical results permit us to draw the following conclusions for each value of $\varepsilon$ :

(1) After a transient phase, the solution of the one-leg method (5.5) approximates the slowly varying solution of (5.1) accurately to one decimal place. The solution of the one-step method (5.6) does not accurately approximate the slowly varying solution of (5.1).

(2) The solution of the one-leg method remains bounded as $\varepsilon \rightarrow 0$. The solution of the one-step method becomes unbounded as $\varepsilon \rightarrow 0$. According to calculations (5.8) and (5.9), the only way to reduce the size of the solution of the one-step method (and to accurately compute the solution or slowly varying solution of (5.1)) is to reduce the step size $k$. Consequently, the one-leg method is more efficient than the one-step method.

Remark 5.2. In order to efficiently solve stiff differential equations, implicit methods are almost always used. The concept of uniform $(k, \varepsilon)$-stability for system (5.1) or (1.4) permits us to compare the efficiency of implicit formulae. That is, an implicit method which is not a uniformly $(k, \varepsilon)$-stable approximation of (5.1) ((1.4)) will be less efficient than an implicit method which is a uniformly $(k, \varepsilon)$-stable approximation of (5.1) ((1.4)).

The tables also illustrate conclusions 1-3 which are stated at the end of Section 3.

Acknowledgment. Several people offered valuable suggestions pertaining to this paper. I would especially like to thank David Gottlieb, Randy LeVeque and the referee for their critical comments. 


\section{APPENDiX I}

\section{Notation.}

$\mathbf{R}^{s}$ denotes $s$-dimensional real Euclidean space.

$\mathbf{C}^{s}$ denotes $s$-dimensional complex space.

$y^{T}$ denotes the transpose of the vector $y$.

$A^{*}$ denotes the conjugate transpose of any matrix $A$.

$\|y\|=\max _{i}\left|y^{(i)}\right|$ denotes the vector norm.

$\|A\|=\sup _{y \neq 0}\|A y\| /\|y\|$ denotes the subordinate matrix norm.

A matrix or vector $f(t, \varepsilon) \in C^{p}(t, B)$ if all derivatives $d^{\nu} f(t, \varepsilon) / d t^{\nu}$ with $0 \leqslant \nu \leqslant p$ are continuous and uniformly bounded by the constant $B$ for $0 \leqslant t \leqslant T$ and $0 \leqslant \varepsilon \leqslant \varepsilon_{0}$, where $\varepsilon_{0}>0$ is a constant.

A matrix or vector $f(t, \varepsilon) \in C^{p}(t, \varepsilon, B)$ if all partial derivatives $\partial^{\nu} f(t, \varepsilon) / \partial t^{\nu_{1}} \partial \varepsilon^{\nu_{2}}$ with $0 \leqslant \nu_{1}+\nu_{2}=\nu \leqslant p$ are continuous and uniformly bounded by the constant $B$ for $0 \leqslant t \leqslant T$ and $0 \leqslant \varepsilon \leqslant \varepsilon_{0}$, where $\varepsilon_{0}>0$ is a constant.

Let $f\left(\varepsilon_{1}, \ldots, \varepsilon_{n}\right)$ and $g\left(\varepsilon_{1}, \ldots, \varepsilon_{n}\right)$ be two vectors or matrices which depend on the $n$ parameters $\varepsilon_{1}, \ldots, \varepsilon_{n}$, and are defined for all $\left(\varepsilon_{1}, \ldots, \varepsilon_{n}\right)$ contained in some set $I$. Then $f\left(\varepsilon_{1}, \ldots, \varepsilon_{n}\right)=O\left(g\left(\varepsilon_{1}, \ldots, \varepsilon_{n}\right)\right)$ for all $\left(\varepsilon_{1}, \ldots, \varepsilon_{n}\right) \in I$ (or just $f\left(\varepsilon_{1}, \ldots, \varepsilon_{n}\right)=$ $O\left(g\left(\varepsilon_{1}, \ldots, \varepsilon_{n}\right)\right)$ when the set $I$ is understood) if there exists a constant $K$, independent of $\varepsilon_{1}, \ldots, \varepsilon_{n}$, so that $\left\|f\left(\varepsilon_{1}, \ldots, \varepsilon_{n}\right)\right\| \leqslant K\left\|g\left(\varepsilon_{1}, \ldots, \varepsilon_{n}\right)\right\|$ for all $\left(\varepsilon_{1}, \ldots, \varepsilon_{n}\right) \in I$.

Division of Applied Mathematics

Brown University

Providence, Rhode Island 02912

1. L. R. Abrahamsson, H. B. Keller \& H.- O. Kreiss, "Difference approximations for singular perturbations of systems of ordinary differential equations," Numer Math., v. 22, 1974, pp. 367-391.

2. G. Buurel, G. Dahlquist, B. Lindberg, S. Linde \& L. Oden, Survey of Stiff Ordinary Differential Equations, Report NA 70.11, Dept. of Information Processing, Royal Institute of Technology, S-100 44 Stockholm 70, Sweden, 1970.

3. K. BurRage, "High order algebraically stable Runge-Kutta methods," BIT, v. 18, 1978, pp. $373-383$

4. K. Burrage \& J. C. Butcher, "Stability criteria for implicit Runge-Kutta methods," SIAM J. Numer. Anal., v. 16, 1979, pp. 46-57.

5. J. C. Butcher, “A stability property of implicit Runge-Kutta methods," BIT, v. 15, 1975, pp. $358-361$

6. M. Crouzeix, "Sur la B-stabilité des méthodes de Runge-Kutta," Numer. Math., v. 32, 1979, pp. $75-82$.

7. (j. G. Dahl.quist, “A special stability problem for linear multistep methods,” BIT, v. 3, 1963, pp. $27-43$.

8. G. G. Dahlquist, On Stability and Error Analysis for Stiff Non-linear Problems, Part 1, Report TRITA-NA-7508, Dept. of Information Processing, Royal Institute of Technology, Stockholm, Sweden, 1975.

9. G. G. Dahlquist, "The sets of smooth solutions of differential and difference equations," in Stiff Differential Sy'stems (R. A. Willoughby, ed.), Plenum Press, New York, 1974, pp. 67-80.

10. G. G. Dahlouist, Some Properties of Linear Multistep and One-leg Methods for Ordinary Differential Equations, Report TRITA-NA-7904, Dept. of Numerical Analysis and Computing Science, Royal Institute of Technology, Stockholm, Sweden, 1979.

11. G. G. Dahlquist \& B. Lindberg, On Some Implicit One-step Methods for Stiff Differential Equations, Report TRITA-NA-7302, Dept. of Information Processing, Royal Institute of Technology, Stockholm, Sweden, 1973. 
12. G. G. Dahlquist \& G. Söderland, Error Propagation in Stiff Differential Systems of Singular Perturhation Type, Report TRITA-NA-8108, Dept. of Information Processing, Royal Institute of Technology, Stockholm, Sweden, 1981.

13. B. L. Ehle, On Padé Approximations to the Exponential Function and A-stable Methods for the Numerical Solution of Initial Value Problems, Dept. of Applied Analysis and Computer Science, University of Waterloo, Research Report No. CSRR 2010, 1969.

14. W. H. ENRIGHT \& T. E. Hull, "Comparing numerical methods for the solution of stiff systems of ODE's arising in chemistry," in Numerical Methods for Differential Systems (L. Lapidus and W. E. Schiesser, eds.), Academic Press, New York, 1976, pp. 45-66.

15. W. H. EnRight, T. E. Hull \& B. LindberG, "Comparing numerical methods for stiff systems of ODE's," BIT, v. 15, 1975, pp. 10-48.

16. S. F. Feschchenko, N. I. Shkil' \& L. D. Nikolenko, Asymptotic Methods in the Theory of Linear Differential Equations, American Elsevier, New York, 1967.

17. R. Frank, J. SChNeid \& C. W. Ueberhuber, “The concept of B-convergence," SiAM J. Numer. Anal., v. 18, 1981, pp. 753-780.

18. C. W. Gear, Numerical Initial Value Problems in Ordinary Differential Equations, Prentice-Hall, Englewood Cliffs, N. J., 1971.

19. A. R. Gourlay, "A note on trapezoidal methods for the solution of initial value problems," Math. Comp., v. 24, 1970, pp. 629-633.

20. F. HopPENSTEADT, "Properties of solutions of ordinary differential equations with small parameters," Comm. Pure Appl. Math., v. 24, 1971, pp. 807-840.

21. F. Hoppensteadt \& W. L. Miranker, Numerical Solution of Differential Equations with Rapidly, Changing Solutions, IBM Report RC4792, IBM Research Center, Yorktown Heights, New York, 1974.

22. H.- O. KreisS, "Difference methods for stiff ordinary differential equations," SIAM J. Numer. Anal., v. 15, 1978, pp. 21-58.

23. H.- O. Kreiss, "Problems with different time scales for ordinary differential equations," SIAM J. Numer. Anal., v. 16, 1979, pp. 980-998.

24. J. D. Lambert, Computational Methods in Ordinary Differential Equations, Wiley, London, 1973.

25. L. LaPidus \& W. E. Schiesser, Numerical Methods for Differential Systems, Academic Press, New York, 1976.

26. B. LindBerg, IMPEX-A Program Package for the Solution of Systems of Stiff Differential Equations, Report TRITA-NA-72.50, Royal Institute of Technology, Stockholm, Sweden, 1972.

27. B. LINDBERG, IMPEX 2-A Procedure for Solution of Systems of Stiff Differential Equations, Report TRITA-NA-7303, Royal Institute of Technology, Stockholm, Sweden, 1973.

28. G. MAJDA, "Filtering techniques for systems of stiff ordinary differential equations I," SIAM J. Numer. Anal., v. 21, 1984, pp. 535-566.

29. G. MAJDA, "Filtering techniques for systems of stiff ordinary differential equations II. Error estimates," SIAM J. Numer. Anal., v. 22, 1985, pp. 1116-1134.

30 G. Majda, A New Theory for Multistep Discretizations of Stiff Ordinary Differential Equations I: Stability with Large Step Sizes, Report NA-3, Division of Applied Mathematics, Brown University, Providence, R.I., 1983.

31. W. L. Miranker, "Numerical methods of boundary layer type for stiff systems of differential equations," Computing, v. 11, 1973, pp. 221-234.

32. W. L. Miranker, Numerical Methods for Stiff Equations and Singular Perturbation Problems, Reidel, Boston, 1981.

33. O. NeVAnlinNa \& W. Liniger, “Contractive methods for stiff differential equations, part 1," $B I T$, v. 18,1978 , pp. $457-474$.

34. F. ODEH \& W. LINIGER, "A note on unconditional fixed- $h$ stability of linear multistep formulae," Computing, v. 7, 1971, pp. 240-253.

35. F. ODeh \& W. Liniger, “Nonlinear fixed $h$-stability of linear multistep formulas,” $J$. Math. Anal. Appl., v. 61, 1977, pp. 691-712.

36. A. Prothero \& A. Robinson, "On the stability and accuracy of one-step methods for solving stiff systems of ordinary differential equations," Math. Comp., v. 28, 1974, pp. 145-162.

37. H. J. StetTer, Towards a Theory for Discretizations of Stiff Differential Systems, in Lecture Notes in Math., vol. 506, Springer-Verlag, New York, 1975, pp. 190-201.

38. H. J. SteTter, Analysis of Discretization Methods for Ordinary Differential Equations, Springer-Verlag, Berlin, 1973.

39. G. W. STRANG, “Accurate partial difference methods II. Non-linear problems," Numer. Math., v. 6 , 1964, pp. $37-46$. 
40. M. van Veldhuizen, Convergence of One-step Discretizations for Stiff Differential Equations, Ph.D. Thesis, Mathematical Institute, University of Utrecht, Netherlands, 1973.

41. M. Van Veldhuizen, "Consistency and stability for one-step discretizations of stiff differential equations," in Stiff Differential Systems (R. Willoughby, ed.), Plenum Press, New York, 1974, pp. 259-270.

42. M. van Veldhuizen, “D-stability,” SIAM J. Numer. Anal., v. 18, 1981, pp. 45-64.

43. W. WAsow, Asymptotic Expansions for Ordinary Differential Equations, Interscience, Wiley, New York, 1965.

44. O. WIDLUND, “A note on unconditionally stable linear multistep methods," $B I T$, v. 7, 1967, pp. $65-70$.

45. R. A. Willoughby, Stiff Differential Systems, Plenum Press, New York, 1974. 


\section{Supplement to Stability with Large Step Sizes for Multistep Discretizations of Stiff Ordinary Differential Equations}

By George Majda

$\underline{\text { APPENDIX II }}$

Proof of Lemma 2.2. (All constants which appear in this proof are independent of $k, t$ and $\varepsilon_{\text {.) }}$

Write $(2.8)$ as its equivalent one-step method

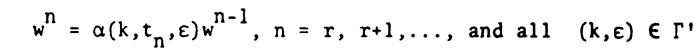

where $w^{n}=\left(y^{n}, y^{n-1}, \ldots, y^{n-r+1}\right)^{T}$ and

$(A .2) \alpha(k, t, \varepsilon)=\left(\begin{array}{ccc}Q_{1}\left(k, \varepsilon, \tau_{0}(t), \ldots, \tau_{r}(t)\right) & \ldots Q_{r}\left(k, \varepsilon, \tau_{0}(t), \ldots, \tau_{r}(t)\right) \\ I & 0 \ldots & . \\ 0 & 10 \ldots & . \\ . & \ldots . & \\ 0 & \ldots I & 0\end{array}\right)$.

Assumption (2.9) implies that $\alpha(k, t, \varepsilon)$ is uniformly bounded for all

$\mathrm{rk} \leq \mathrm{t}<\infty$ and $(k, \varepsilon) \in \mathrm{r}^{\prime}$.

Assumptions (2.9) and (2.10) imply that for all rk $\leq t<\infty$ and $(k, \varepsilon) \in \Gamma^{\prime}$

(A.3)

$\alpha(k, t, \varepsilon)=B(k, t, \varepsilon)+k \gamma(k, t, \varepsilon)$

with 

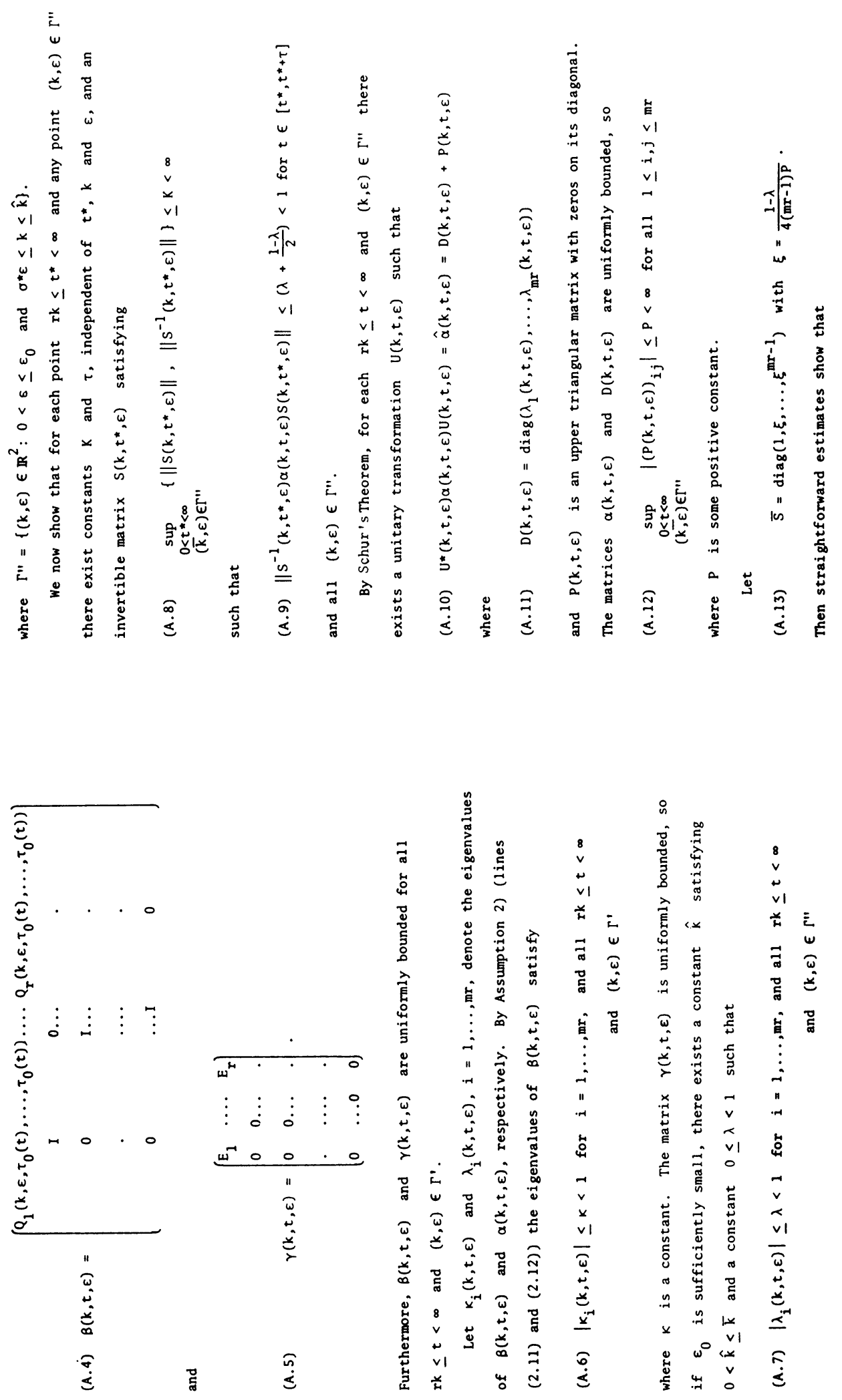

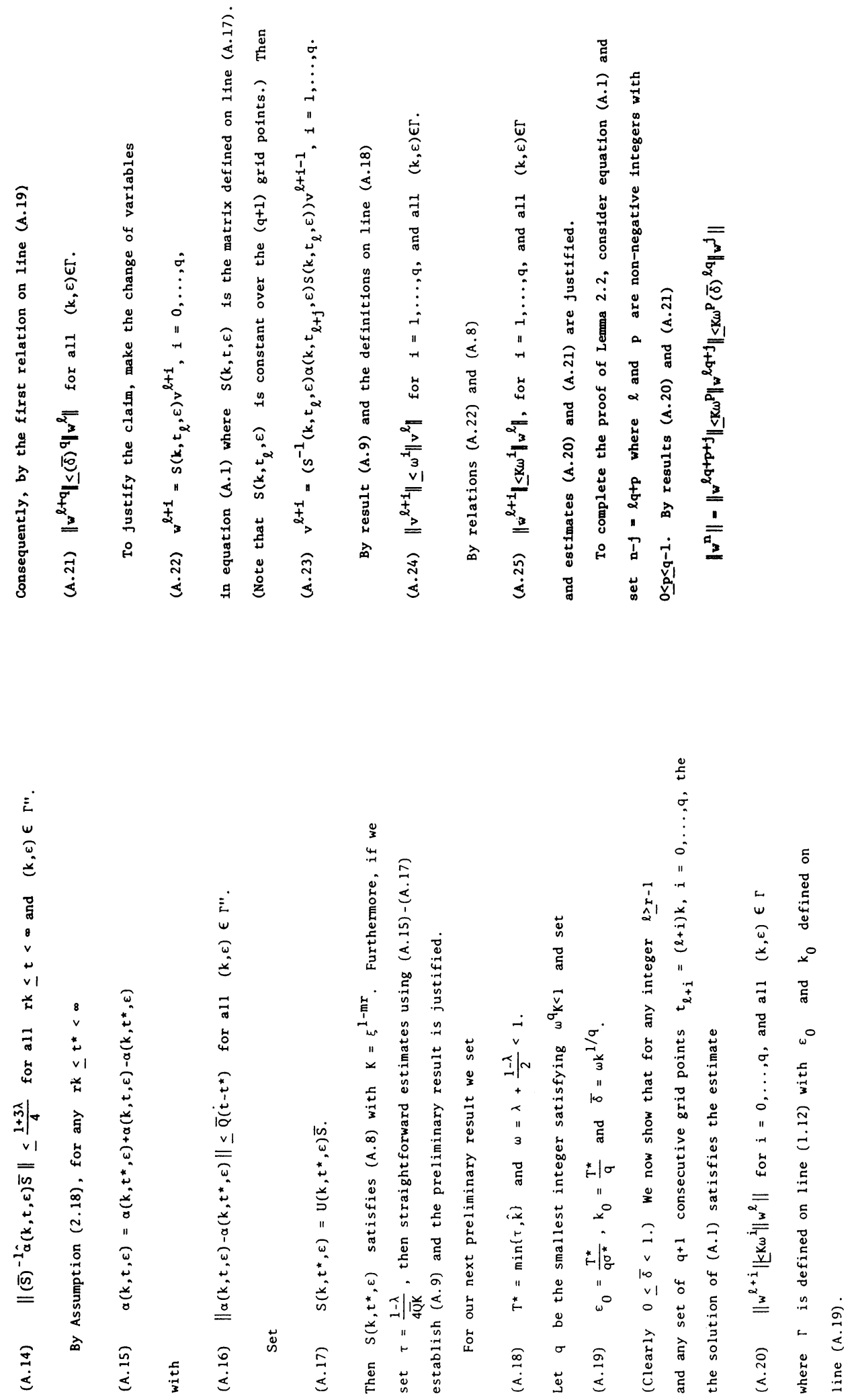


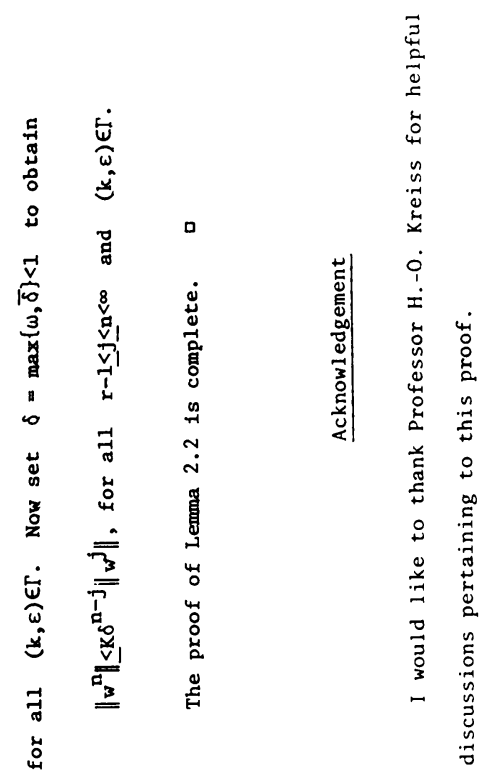

